\title{
Analyticity and the Daughter Structure of Conspiring Regge-Pole Families
}

\author{
LORELLA JONES* ${ }^{*}$ \\ California Institute of Technology, Pasadena, California 91109 \\ AND \\ HARVEY K. SHEPARD $\ddagger$ \\ University of California, Riverside, California 92502 \\ (Received 20 May 1968)
}

\begin{abstract}
By studying reactions involving unequal-mass particles with spin, we show that much of the structure of possible families of conspiring Regge poles follows simply from imposing the $t=0$ analyticity constraints. These requirements imply the necessity for both daughter and conspirator contributions, where in many cases the daughter poles are themselves conspirators. We discuss the pattern of $(t=0)$ singularity cancellation by daughter trajectories in both the single-parity families and double-parity families for general mass and spin processes and demonstrate that the two have rather different structure. As an application, the reactions $\pi \pi \rightarrow V V, \pi N \rightarrow V N$, and $N N \rightarrow N N$ are examined in detail from the point of view of analyticity constraints. We show that in all cases factorization of the (first) daughter residues is consistent with the required analyticity properties of the amplitudes, and that in certain (nonevasive) cases the factorization of the daughter residues is directly implied by factorization for the leading pole when the conspiracy constraints are obeyed. We conclude that our results, based on analyticity (and factorization), complement the grouptheoretic $O(4)$ classification; the symmetry and analyticity methods give similar information when they overlap, but supplement each other in certain cases when one method is not readily applicable.
\end{abstract}

\section{INTRODUCTION}

$\mathrm{D}^{4}$ AUGHTER trajectories were introduced by Freedman and Wang ${ }^{1}$ into the treatment of unequalmass spinless scattering to preserve the analyticity of Reggeized amplitudes at $t=0$. They and others ${ }^{2-4}$ pointed out that the existence both of daughter poles in unequal-mass spinless scattering and conspirator trajectories in the case of equal-mass scattering with spin can be inferred from an analysis based on considerations of the Lorentz group $O(3,1)$. Several classes or types of conspiring families of poles were isolated, essentially only those which can couple to the $N \bar{N}$ system. Domokos ${ }^{5}$ has argued that the classification of the spectrum of Regge poles into $O(3,1)$ families, where each family corresponds to a single Lorentz or Toller pole, ${ }^{3}$ is independent of the external masses in the problem, and thus applies to unequal-mass scattering as well.

By studying a more general case containing both unequal masses and spins, we show that much of the

\footnotetext{
* Work supported in part by the U. S. Atomic Energy Commission. Prepared under Contract AT(11-1)-68 for the San Francisco Operations Office, U. S. Atomic Energy Commission.

$\dagger$ Present address: Physics Department, University of Illinois, Urbana, Ill.

$\ddagger$ Work supported in part by the U. S. Atomic Energy Commission. Prepared under Contract AT(11-1)-34P107A for the San Francisco Operations Office, U. S. Atomic Energy Commission.

${ }_{1}$ D. Z. Freedman and J. M. Wang, Phys. Rev. Letters 17, 569 (1966); Phys. Rev. 153, 1596 (1967).

${ }_{2}$ G. Domokos and P. Suranyi, Nucl. Phys. 54, 529 (1964).

${ }^{3}$ M. Toller, Nuovo Cimento 37, 631 (1965); 53A, 671 (1968); University of Rome Internal Reports Nos. 76 and 84, 1965 (unpublished); CERN Reports Nos. Th770 and Th780, 1967 (unpublished); L. Sertorio and M. Toller, Nuovo Cimento 33, 413 (1964). ${ }^{4}$ D. Z. Freedman and J. M. Wang, Phys. Rev. Letters 18, 863 (1967); Phys. Rev. 160, 1560 (1967).

${ }^{5}$ G. Domokos, Phys. Rev. 159, 1387 (1967); G. Domokos and G. Tindle, ibid. 165, 1906 (1968).
}

detailed structure of possible conspiring families of Regge poles follows simply from imposing the $t=0$ analyticity constraints. These constraints on helicity amplitudes are of two types: (1) restrictions on the analytic structure of individual parity-conserving helicity amplitudes (PCHA) as analyzed by $\mathrm{Hara}^{6}$ and Wang $^{7}$; and (2) the conspiracy equations, which are linear constraints between different helicity amplitudes (at $t=0) .{ }^{8-11}$ Such analyticity requirements (in the case of unequal-mass scattering with spin) imply the necessity for both daughter and conspirator contributions. ${ }^{12}$ Although the work of Ref. 1 makes it plausible, it is not obvious for general mass and spin processes that the necessary daughter-conspirator contributions can be supplied by a single Lorentz pole. We demonstrate explicitly that, once the parent pole(s) has (have) been specified, all $t=0$ constraints may be satisfied by including the daughter sequence.

Thus we show that analyticity induces the same Regge-pole family structure as that given by the $O(3,1)$ group-theoretic approach. This analyticity point of view makes it easy to investigate some types of ( $M=0$ and $M=1$ ) conspiracy not previously discussed. It also

\footnotetext{
${ }^{6}$ Y. Hara, Phys. Rev. 136, B507 (1964).
}

${ }^{7}$ L. L. Wang, Phys. Rev. 142, 1187 (1966); 153, 1664 (1967).

${ }^{8} \mathrm{D}$. V. Volkov and V. N. Gribov, Zh. Eksperim. i Teor. Fiz. 44, 1068 (1963) [English transl.: Soviet Phys.-JETP 17, 720 (1963)].

${ }_{9}$ M. Gell-Mann and E. Leader, in Proceedings of the Thirteenth International Conference on High-Energy Physics, Berkeley, 1966

(University of California Press, Berkeley, Calif., 1967).

${ }_{10}$ E. Leader, Phys. Rev. 166, 1599 (1968).

${ }^{11}$ G. Cohen-Tannoudji, A. Morel, and H. Navelet, Ann. Phys. (N. Y.) 46, 239 (1968).

${ }_{12}$ During the final stages of preparation of this work, we received a paper by M. A. Jacobs and M. H. Vaughn, Phys. Rev. 172, 1677 (1968), which discusses some of the same questions from a slightly different point of view. 
allows the phenomenologist interested in fitting highenergy data to simply construct formulas which are correct in all kinematic details. We have assembled enough details to allow one to construct appropriate formulas for any mass case, with Lorentz-pole quantum number $M=0$ or $M=1$. These formulas have the expected property that (because daughters have been included) the leading term in the Regge expansion dominates at high energies for inelastic as well as elastic processes for all values of the momentum transfer. (Some coefficients of secondary powers of $s$ have, however, heretofore unexpected singularities near $t=0$.)

There are four conceivable families consisting of a single leading pole, and all are similar in structure from the point of view of analyticity. Only two of these have previously been discussed (in Ref. 4). In unequal-mass scattering, given the leading (parent) pole, the existence of odd and even daughters is required by the analyticity of individual PCHA. In $O(3,1)$ language this would be saying that analyticity ensures that the entire Toller ${ }^{3}$ pole contributes to the amplitude if the leading member does. (When the parent trajectory intersects $t=0$ at an integer, only a finite number of daughters are required.) For each amplitude, the most singular part of the daughter residues is determined by requiring a cancellation of unwanted singularities. For equal-mass scattering, daughters are not required in order to satisfy the analyticity requirements on individual PCHA. Thus, for this case the singularity-cancellation method gives us no information about possible families of poles (although if we wish to have a nonevasive solution to the conspiracy relations, daughters are necessary in some cases). However, by imposing residue factorization to link the equal- and unequal-mass processes, the equalmass daughter residues can usually be determined. Analyticity plus factorization thus provide a great deal of information.

The daughter trajectories in this single-parity type of family may also be conspirators. ${ }^{13}$ The two single-parity families considered by Freedman and Wang in their treatment of $N N$ scattering ${ }^{4}$ differ in that the odd daughters of a class-I family [with $P=C=(-1)^{J}$ leading pole] cannot couple to the $N \bar{N}$ system; and thus only the class-II family [with $P=C=-(-1)^{J}$ leading pole] allows a nonevasive solution, with the (odd) daughters becoming conspirators. We show that the two $M=0$ families not treated in Ref. 4 do not couple to $N \bar{N}$ at $t=0$ although there are other systems where they may (and probably do) contribute. (The ordinary physical pion may, in fact, belong to one of these classes.)

The detailed structure of families with parity-doubled leading poles is rather different from that of the singleparity families. Such families appear only when one studies reactions involving external particles with suffi-

\footnotetext{
${ }^{13}$ B. Diu and M. Le Bellac, Nuovo Cimento 53A, 158 (1968).
}

ciently high $\operatorname{spin}^{14}$; for spinless scattering the singleparity families of daughters are all that is needed to preserve the desired analyticity. We find, in a paritydoubled family, a succession of parity-doubled daughters. The daughters of each parity cancel singularities arising from contributions due to both parities. There are two $M=1$ families, differing in charge conjugation; one of these does not couple to $N \bar{N}$ at $t=0$ (this one had been ignored previously). ${ }^{4}$ The pattern of $t=0$ singularity cancellation is not what one would obtain from the superposition of two single-parity $M=0$ families.

In addition to a discussion of daughters in general mass and spin processes, the present paper contains a detailed examination of the reactions $\pi \pi \rightarrow V V, \pi N \rightarrow$ $V N$, and $N N \rightarrow N N$ from the point of view of analyticity constraints. This analysis supplements and clarifies recent work $^{15-17}$ on these processes, which are coupled by the requirements of Regge-residue factorization. Besides giving a concrete illustration of our approach, the residues we determine are useful in treating recent forward inelastic experiments (e.g., $\pi N \rightarrow \rho N)$ and in the discussion of the $O(4) M$ quantum number of the pion.

We also demonstrate for certain cases that factorization of the residue of the parent Regge pole implies factorization of the residue of the first daughter contribution, when the conspiracy constraints are obeyed. For other cases, factorization of the first daughter residues is consistent with all analyticity constraints, but cannot be demonstrated a priori. Our work leads us to believe that in general all daughters derived from analyticity are consistent with factorization. Factorization, moreover, is enormously useful because it links equal- and unequal-mass reactions. It thus removes much of the (unwanted) freedom left after all analyticity requirements are satisfied.

In Sec. II we define the properties of "daughter" trajectories and demonstrate why daughters are required to preserve the $t=0$ analyticity of individual Reggeized helicity amplitudes. We give the expansions of parent- and daughter-pole contributions which form the basis of our later discussion. These are an extension of the well-known treatment of daughters in the spinless case. ${ }^{1}$ In this section we indicate the pattern of singularity cancellation for both single- and doubleparity families and describe the $t=0$ behavior of the successive residues. Section III introduces conspiracy, the other analyticity constraint at $t=0$, and gives the conspiracy relations between PCHA for the specific processes we are considering. In Sec. IV, we show how the analyticity constraints studied in Secs. II and III are

${ }^{14}$ If the exchanged pole gives a finite contribution at $t=0$, it belongs to an $O(4)$ family with $M \leq \min \left(S_{i}, S_{f}\right)$ where $S_{i(f)}$ is the total spin of the initial (final) $t$-channel state. See R. F. Sawyer, Phys. Rev. 167, 1372 (1968). Also see M. Toller, Ref. 3, and S. A. Klein, Claremont College Report (unpublished).

${ }_{15}$ L. Jones, Phys. Rev. 163, 1523 (1967).

$16 \mathrm{~S}$. Frautschi and L. Jones, Phys. Rev. 167, 1335 (1968).

${ }^{17}$ H. Shepard, Phys. Rev. 168, 1572 (1968). 
satisfied by families with a single leading pole, and thus illustrate how daughter poles may be conspirators. The contribution of families with parity-doubled leading trajectories to these reactions is discussed in Sec. V. In Sec. VI we compare our results, based on analyticity, with the group-theoretic $O(3,1)$ classification.

Supplementary material is presented in the appendices: Appendix A summarizes the functions which we use to expand the PCHA; Appendices B and C are devoted to proofs that for the case of one leading trajectory a single set of daughters removes singularities from both "dominant" and "minor" PCHA. Examples from perturbation theory, which we have found very helpful, are collected in Appendix D.

\section{DAUGHTERS AND THE REGGE-POLE EXPANSION OF (INDIVIDUAL) PARITY- CONSERVING HELICITY AMPLITUDES}

In this section we wish to give most of the kinematic details and techniques which will be (implicitly) used in the remainder of the paper. Principally, we shall show how parent and daughter poles contribute to the expansions of the Reggeized PCHA for general spins and masses. By demonstrating the way in which daughters remove singularities from the coefficients of certain powers of $s$ in the Regge expansion, we generalize the discussion given in Ref. 1 for spinless unequal-mass scattering. The technique of singularity cancellation presented in this section will be useful in other applications besides those given in the present paper.

\section{A. Preliminary Kinematics}

We define the $s$ channel to be the reaction

the $t$ channel is

$$
a+b \rightarrow c+d
$$

$$
D^{\prime}\left(\lambda_{1}\right)+b^{\prime}\left(\lambda_{2}\right) \rightarrow c^{\prime}\left(\lambda_{3}\right)+A^{\prime}\left(\lambda_{4}\right)
$$

where we have written the helicity of each $t$-channel particle in parentheses. The $t$-channel helicity amplitudes will be written $f_{34,12}(s, t)$ and we define ${ }^{18}$

$$
\bar{f}_{34,12}=\left(\sqrt{2} \cos \frac{1}{2} \theta_{t}\right)^{-|\lambda+\mu|}\left(\sqrt{2} \sin \frac{1}{2} \theta_{t}\right)^{-|\lambda-\mu|} f_{34,12},
$$

where $\lambda=\lambda_{1}-\lambda_{2}, \mu=\lambda_{3}-\lambda_{4}$. The $\bar{f}_{34,12}$ are assumed to have only dynamical singularities in $s$; they have the partial-wave expansion $\tilde{f}_{34,12}=\sum(2 J+1) T_{34,12}{ }^{J} e_{\lambda \mu} J$. The functions $e_{\lambda \mu}{ }^{J}$ are defined in Appendix A.

When $m_{a}=m_{c}$ and $m_{b}=m_{d}$, we have elastic $s$-channel scattering. The initial and final $t$-channel states each consist of an equal-mass pair; we call this case EE scattering. Similarly, we describe the case $m_{a}=m_{c}$ and $m_{b} \neq m_{d}$ as EU scattering, and the case $m_{a} \neq m_{c}$ and $m_{b} \neq m_{d}$ as UU scattering.

${ }^{18}$ M. Gell-Mann, M. Goldberger, F. Low, E. Marx, and F. Zachariasen, Phys. Rev. 133, B145 (1964).
The $t$-channel center-of-mass momenta are

$$
\begin{array}{r}
q \equiv p_{D b}=\left[t-\left(m_{d}+m_{b}\right)^{2}\right]^{1 / 2}\left[t-\left(m_{d}-m_{b}\right)^{2}\right]^{1 / 2} / 2 \sqrt{ } t \\
\equiv T_{D b} / 2 \sqrt{ } t \\
p \equiv p_{c A}=\left[t-\left(m_{a}+m_{c}\right)^{2}\right]^{1 / 2}\left[t-\left(m_{a}-m_{c}\right)^{2}\right]^{1 / 2} / 2 \sqrt{ } t \\
\equiv T_{A c} / 2 \sqrt{ } t
\end{array}
$$

and

$$
\begin{aligned}
\cos \theta_{t} & =z_{t}=z=\left(2 s t+t^{2}-t \Sigma+\Delta_{d b} \Delta_{c a}\right)\left(T_{a c} T_{d b}\right)^{-1} \\
& =\left(2 s+t-\Sigma+\Delta_{d b} \Delta_{c a} / t\right)(4 p q)^{-1} \\
& =\left(\nu+\Delta_{d b} \Delta_{c a} / t\right)(4 p q)^{-1}
\end{aligned}
$$

where

$$
\begin{gathered}
\Sigma=m_{a}^{2}+m_{b}^{2}+m_{c}^{2}+m_{d}^{2}, \quad \Delta_{i j}=m_{i}{ }^{2}-m_{j}{ }^{2}, \\
\nu=s-u=2 s+t-\Sigma .
\end{gathered}
$$

The following will be useful:

$(p q z)^{\alpha}=4^{-\alpha}\left(\nu+\Delta_{d b} \Delta_{c a} / t\right)^{\alpha}=4^{-\alpha} \nu^{\alpha}\left(1+\Delta_{d b} \Delta_{c a} / \nu t\right)^{\alpha}$.

The last factor is equal to 1 for EE or EU scattering.

Study of the large- $s$, small- $t$ region makes it convenient to expand the powers of $\cos \theta_{t}$ as follows:

$$
\begin{aligned}
& {\left[\nu+\Delta_{d b} \Delta_{c a} / t\right]^{\alpha} \equiv\left(\nu^{\prime}\right)^{\alpha}=\nu^{\alpha}\left(1+\Delta_{d b} \Delta_{c a} / \nu t\right)^{\alpha}} \\
& \underset{s \rightarrow \infty}{\rightarrow} \nu^{\alpha}+\alpha \Delta_{d b} \Delta_{c a} t^{-1} \nu^{\alpha-1}+\frac{1}{2} \alpha(\alpha-1)\left(\Delta_{d b} \Delta_{c a}\right)^{2} t^{-2} \nu^{\alpha-2}+\cdots \\
& \quad=\sum_{n=0}\left(\begin{array}{l}
\alpha \\
n
\end{array}\right)\left(\Delta_{d b} \Delta_{c a} / t\right)^{n} \nu^{\alpha-n},
\end{aligned}
$$

where (near $t=0$ )

$$
\begin{aligned}
\nu^{\alpha} & =(2 s+t-\Sigma)^{\alpha} \rightarrow 2^{\alpha} s^{\alpha}(1-\Sigma / 2 s)^{\alpha} \\
& =2^{\alpha} \sum_{l=0}\left(\begin{array}{l}
\alpha \\
l
\end{array}\right)(-\Sigma / 2)^{l} s^{\alpha-l} \\
& =2^{\alpha}\left[s^{\alpha}-\frac{1}{2} \alpha \Sigma s^{\alpha-1}-\frac{1}{8} \alpha(\alpha-1) \Sigma^{2} s^{\alpha-2}+\cdots\right] .
\end{aligned}
$$

If $\alpha$ is an integer, the expansion eventually terminates.

We form Reggeized parity-conserving helicity amplitudes (PCHA) according to the method of Ref. 18. (Also see Ref. 7.) Examples will be given shortly. The complete residue of the Regge-pole $(\alpha)$ contribution to the partial-wave (definite-parity) amplitude $T_{34,12}{ }^{J P}$ $=\frac{1}{2}\left(T_{34,12}^{J} \pm T_{-3-4,12}{ }^{J}\right)$ will be written

$$
\beta_{34,12}^{P C}(t)=\beta_{\mu \lambda}{ }^{P C}(t)=(2 \alpha+1) K(t) \gamma(t)(p q)^{\alpha-\Lambda},
$$

where $\Lambda=\max (|\lambda|,|\mu|)$ and the residue is defined by $(2 \alpha+1) T_{34,12}{ }^{\alpha P} \rightarrow \beta / J-\alpha$ at the Regge pole in the Sommerfeld-Watson transform of the PCHA

$$
\begin{aligned}
\bar{f}_{34,12}{ }^{P C}=\sum(2 J & +1) \frac{1}{2}\left(T_{34,12}{ }^{J} \pm T_{-3-4,12}\right) e_{\lambda \mu}{ }^{J^{+}} \\
& +\sum(2 J+1) \frac{1}{2}\left(T_{34,12}{ }^{J} \mp T_{-3-4,12}{ }^{J}\right) e_{\lambda \mu}{ }^{J-} .
\end{aligned}
$$

The superscripts $P C$ refer to the parity and chargeconjugation behavior of the exchanged Regge pole in the $t$ channel (assumed to be a meson); this can be described by specifying $P, C= \pm(-1)^{J}$, or just \pm for brevity. The factor $K(t)$ contains the kinematic singu- 
larities and zeros of the one PCHA with the quantum numbers of the exchange; these can be isolated using the rules given by Wang. ${ }^{7}$ Notice that when $\lambda$ and $\mu$ are both nonzero, once $\beta_{\lambda \mu}{ }^{P C}(t)$ is calculated for the PCHA with "dominant parity" $P$, the contribution of this Regge pole to the PCHA with the same $\lambda, \mu$ but with "dominant parity" $-P$ is completely determined as

$$
\beta_{\lambda \mu}^{P C} e_{\lambda \mu}{ }^{J}\left[1 \pm e^{-i \pi \alpha}\right] / \sin \pi \alpha .
$$

The Wang $K^{-P}(t)$ for this "minor" amplitude agrees with the net singularity of $K^{+P}(t)(p q)^{\alpha-\Lambda} e_{\lambda \mu}{ }^{\alpha^{-}}$; hence the formalism is consistent in this regard. ${ }^{19}$ (See further discussion of this point later.)

The last factor in $\beta(t)$, viz., $(p q)^{\alpha-\Lambda}$, can be derived from the Froissart-Gribov expression for the partialwave amplitude. ${ }^{20}$ As $\beta(t)$ thus defined is $(2 \alpha+1)$ times the residue of the partial-wave amplitude, we require the full $\beta(t)$ to satisfy factorization for exchange of poles with definite quantum numbers. Generally, we will not be concerned with the $\alpha$-dependent factors in the residue-e.g., those due to sense-nonsense considerations.

We shall assume the reader is familiar with Appendix A of Ref. 17, which contains a summary of the parity and charge-conjugation properties of PCHA. For the purposes of this paper, we would simply like to recall a few particulars:

(i) If $\lambda \neq 0$ and $\mu \neq 0$, the PCHA receive contributions from states with both kinds of parity, $P= \pm(-1)^{J}$.

(ii) For a boson-antiboson state in which the particle is not its own antiparticle, the states of definite parity will also have definite $C$ when $\lambda_{1}= \pm \lambda_{2}$.

For a boson-antiboson state in which the particle is its own antiparticle, each helicity state will be an eigenstate of $C$ with eigenvalue + :

$$
C\left|J \lambda_{1} \lambda_{2}\right\rangle=+\left|J \lambda_{1} \lambda_{2}\right\rangle \text {. }
$$

Hence, each state of definite parity will have definite $C=+$, and only exchanges with $C=+$ at points with physical signature can contribute. Additional information can be obtained from the requirement of Bose statistics ${ }^{21}$ : If $\lambda_{1}=\lambda_{2}$, only even $J$ will have a nonvanish-

${ }^{19}$ That is, the same type of singularities are present. If only one pole is contributing, $\gamma(t)$ may be forced, by the behavior of the "minor" amplitude, to have additional zeros.

${ }^{20}$ To establish this behavior for the residue, $\beta \sim(p q)^{\alpha-\Lambda}$, one must consider the $t$-channel thresholds and pseudothresholds, $t=\left(m_{1} \pm m_{2}\right)^{2},\left(m_{3} \pm m_{4}\right)^{2}$, and also (for EU or UU) the point $t=0$. In the present work it is the $t=0$ dependence which is of most interest; for there the relation implies [neglecting other $t$ dependence included in the factors $K(t)$ and $\gamma(t)$ in Eq. (2.8)]: (a) $\beta \sim\left(t^{-1 / 2}\right)^{\alpha(0)-\Lambda}$ for EU scattering, and (b) $\beta \sim\left(t^{-1}\right)^{\alpha(0)-\Lambda}$ for UU scattering. This result is therefore a generalization of the behavior of the partial-wave amplitude, $u^{-\alpha(0)}$, derived by Freedman and Wang (Ref. 1) for backward (spinless) $\pi N$ scattering. Following the procedure of Ref. 1, one may construct a similar proof for scattering with spin (with Jacobi polynomials replacing ordinary Legendre polynomials). It is perhaps worth noting that daughters are required in order to establish this $t=0$ behavior, and also that the resulting $t$ dependence plays an important role in the factorization conditions for unequal-mass processes.

${ }^{21}$ M. Jacob and G. C. Wick, Ann. Phys. (N. Y.) 7, 404 (1959). ing contribution, and if $\lambda_{1}=-\lambda_{2}$, only states with $P=(-1)^{J}$ can couple.

(iii) In UU scattering, the PCHA has in general no definite behavior under charge conjugation, and trajectories with either relation of $C$ to $J$ may be exchanged for each parity.

If it should happen that each of the particles at a UU vertex is its own antiparticle, the helicity amplitudes will transform like $C\left|J \lambda_{1} \lambda_{2}\right\rangle=\eta_{1}{ }^{c} \eta_{2}{ }^{c}\left|J \lambda_{1} \lambda_{2}\right\rangle$. Again, only exchanges with $C=\eta_{1}{ }^{c} \eta_{2}{ }^{c}$ at points of physical signature will be allowed. As the particles are not identical, there are no restriction from statistics.

(iv) If the fermion-antifermion state in $\mathrm{EU}$ or $\mathrm{EE}$ scattering has $\lambda_{3} \neq \pm \lambda_{4}$, the PCHA also has no definite behavior under charge conjugation.

(v) When $\lambda_{3}= \pm \lambda_{4}$ for the $F \bar{F}$ state, each parity part of the PCHA has a definite $C$ as well. For $N \bar{N}$ we always have $\lambda_{3}= \pm \lambda_{4}$.

\section{B. Daughters}

Since we are not proceeding from a group-theoretic point of view, we must define what we mean by daughter poles. All discussion here is based on analyticity requirements at $t=0$; the concept of daughters used below is principally of value at that point and we shall not attempt to generalize it to $t \neq 0$.

In order that they contribute to the same processes, all daughters of a parent or leading pole must have the same internal quantum numbers (baryon number, isospin, hypercharge, charge conjugation) as their parent. We choose the daughters to have angular momentum at $t=0$ spaced by integers from that of the parent so that they have the possibility of cancelling the singular coefficients which arise when we make Regge-pole expansions of the PCHA. In order that parent and daughter contributions have the same phase at this point, we shall assume that the odd-numbered daughters (those at $\alpha-1, \alpha-3, \alpha-5$, etc.) have opposite signature from the parent pole, while the even daughters have the same signature as the parent.22

When there is a single parent pole, we insist that the daughters have the same relation of $P$ to $J$ as the parent (e.g., a $1^{-}$parent would have a $0^{+}$daughter); this ensures that they would contribute to the same UU spinless processes and would play the role there outlined by Freedman and Wang. ${ }^{1}$ In order to have the same intrinsic charge-conjugation quantum number at $t=0$, the daughters must correct for their spacing in the definition of $C= \pm(-1)^{J}$. That is, the $n$th daughter must have $C_{(n)}=(-1)^{n} C_{\text {parent }}$. Summarizing, if $P$ and $C$ are the parity and charge conjugation of the parent as given by $\pm(-1)^{J}$, then the odd daughters have $P_{\text {(odd) }}=P, C_{\text {(odd) }}=-C$, and the even daughters have $P_{\text {(even) }}=P, C_{(\text {even })}=C$.

${ }^{22}$ This choice of signature is in accord with the assumption normally made in Regge-pole theory that the phase below threshold is completely determined by signature and kinematic factors. 


\section{Regge-Pole Expansion of PCHA}

We shall employ the expansions of the $e_{\lambda \mu}{ }^{\alpha \pm}(z)$ functions $^{18}$ given in Appendix $\mathrm{A}$ and sometimes choose specific values of $\lambda$ and $\mu$ to illustrate the method. As examples which will be useful in Secs. IV and V, we present the cases (a) $\lambda=0, \mu=1$, where (because $e_{\lambda \mu}{ }^{\alpha-}$ $=0$ when $\lambda=0$ or $\mu=0$ ) only one parity enters, and (b) $\lambda=1, \mu=1$, where Regge poles of both parities can contribute to the PCHA. Given the expansion of $e_{\lambda \mu}{ }^{\alpha \pm}$, any other case can be done in the same manner.

We shall write the Regge-pole contribution to the PCHA

$$
\bar{f}_{34,12}^{ \pm \eta}=\bar{f}_{34,12} \pm(-1)^{\lambda+\Lambda} \eta_{3} \eta_{4}(-1)^{S_{3}+S_{4}} \bar{f}_{-3-4,12}
$$

in the form

$$
\begin{aligned}
\bar{f}_{34,12}{ }^{\eta} & \rightarrow \beta_{34,12}{ }^{R}\left[\left(e^{-i \pi \alpha_{R}}+\tau_{R}\right) / \sin \pi \alpha_{R}\right] e_{\lambda \mu}{ }^{\alpha R^{+}}(z) \\
& +\beta_{34,12}{ }^{Q}\left[\left(e^{-i \pi \alpha_{Q}}+\tau_{Q}\right) / \sin \pi \alpha_{Q}\right] e_{\lambda \mu}{ }^{\alpha Q^{-}}(z),
\end{aligned}
$$

where the poles $\alpha_{R}$ and $\alpha_{Q}$ have opposite $P$. To obtain $\bar{f}^{-\eta}$, we interchange $R$ and $Q$ in (2.10); i.e., $\bar{f}_{34,12}{ }^{-\eta}$ $=\bar{f}_{34,12} \eta(R \leftrightarrow Q)$. We call the parity of the pole associated with the $e^{\alpha+}$ term the "dominant" parity and the parity of the pole in the $e^{\alpha-}$ term the "minor" parity, for reasons which will become obvious. Substituting Eq. (2.8) for the residues, we have

$$
\begin{aligned}
& \bar{f}_{34,12}{ }^{\eta}=\bar{f}_{\mu \lambda}{ }^{\eta} \rightarrow\left(2 \alpha_{R}+1\right) K_{\mu \lambda}{ }^{R}(t) \gamma_{\mu \lambda}{ }^{R}(t) \chi^{R}(\tau) \\
& \times(p q)^{\alpha R^{-\Lambda}} e_{\lambda \mu}{ }^{\alpha R^{+}}(z)+\left(2 \alpha_{Q}+1\right) K_{\mu \lambda}{ }^{Q}(t) \\
& \times \gamma_{\mu \lambda}{ }^{Q}(t) \chi^{Q}(\tau)(p q)^{\alpha Q^{-\Lambda}} e_{\lambda \mu}{ }^{\alpha Q^{-}}(z),
\end{aligned}
$$

where

$$
\chi(\tau) \equiv\left(e^{-i \pi \alpha}+\tau\right) / \sin \pi \alpha
$$

The functions $e_{\lambda \mu}^{\alpha \pm}(z)$ have simple expansion in $z$. Symbolically,

$$
\begin{aligned}
& e_{\lambda \mu}^{\alpha+}(z)=A_{0} z^{\alpha-\Lambda}+A_{2} z^{\alpha-\Lambda-2}+A_{4} z^{\alpha-\Lambda-4}+\cdots \\
& e_{\lambda \mu}^{\alpha-}(z)=A_{1} z^{\alpha-\Lambda-1}+A_{3} z^{\alpha-\Lambda-3}+A_{5} z^{\alpha-\Lambda-5}+\cdots
\end{aligned}
$$

For $\alpha$ nonintegral, these expansions do not terminate. As mentioned before, for $\lambda$ or $\mu=0, e^{\alpha-}=0$, and only the dominant-parity contribution to the PCHA remains. Notice that $e^{\alpha+}$ and $e^{\alpha-}$ contain different powers of $z$. This means that when they are multiplied by the same residue functions, as in $\bar{f}^{\eta}$ and $\bar{f}^{-\eta}$, the resulting amplitudes will have different kinematic singularities at $t=0$ and at threshold (as required from crossing-matrix considerations $\left.{ }^{6,7}\right)$. Stated another way, the threshold singularity of the total contributions of minor- and dominant-parity types to a given PCHA is the same even though the threshold behavior of the partial-wave amplitudes for the opposite-parity cases must be different. As it is generally easiest to think in terms of the dominant contributions, we get $K^{R}(t)$ from $\bar{f}^{+\eta}$ and $K^{Q}(t)$ from $\bar{f}^{-\eta}$, using the rules of Ref. 7 .

Using Appendix A, we have (assuming for the moment only one pole of each parity)

$$
\begin{aligned}
& \bar{f}_{10}{ }^{\eta} \rightarrow(2 \alpha+1) K_{10} \gamma_{10} \chi(p q)^{\alpha-1} e_{01}{ }^{\alpha+}(z) \\
& \rightarrow K_{10} \gamma_{10} \chi\left(\frac{2^{\alpha}(2 \alpha+1)}{[\alpha(\alpha+1)]^{1 / 2}} \frac{\Gamma(\alpha+1 / 2)}{\Gamma(\alpha+1)}\right)\left(\alpha(p q z)^{\alpha-1}-\frac{\alpha(\alpha-1)(\alpha-2)(p q)^{2}(p q z)^{\alpha-3}}{2(2 \alpha-1)}+\cdots\right), \\
& \bar{f}_{11^{\eta}} \rightarrow\left(2 \alpha_{R}+1\right) K_{11}^{R} \gamma_{11}^{R} \chi^{R}(p q)^{\alpha^{R-1}} e_{11}{ }^{\alpha^{+}}(z)+\left(2 \alpha_{Q}+1\right) K_{11}{ }^{Q} \gamma_{11}{ }^{Q} \chi^{Q}(p q)^{\alpha^{Q-1}} e_{11^{\alpha Q^{-}}}(z) \\
& \rightarrow K_{11}{ }^{R} \gamma_{11}{ }^{R} \chi^{R}\left(\frac{2^{\alpha_{R}}\left(2 \alpha_{R}+1\right)}{\alpha_{R}\left(\alpha_{R}+1\right)} \frac{\Gamma\left(\alpha_{R}+1 / 2\right)}{\Gamma\left(\alpha_{R}+1\right)}\right)\left\{(p q z)^{\alpha_{R}-1} \alpha_{R}{ }^{2}-(p q)^{2}(p q z)^{\alpha_{R}-3} \frac{\alpha_{R}\left(\alpha_{R}-1\right)\left(\alpha_{R}-2\right)^{2}}{2\left(2 \alpha_{R}-1\right)}+\cdots\right\} \\
& +K_{11}^{Q} \gamma_{11}{ }^{Q} \chi Q\left(-\frac{2^{\alpha Q}\left(2 \alpha_{Q}+1\right)}{\alpha_{Q}\left(\alpha_{Q}+1\right)} \frac{\Gamma\left(\alpha_{Q}+1 / 2\right)}{\Gamma\left(\alpha_{Q}+1\right)}\right) p q\left\{(p q z)^{\alpha_{Q}-2} \alpha_{Q}\left(\alpha_{Q}-1\right)-(p q)^{2}(p q z)^{\alpha_{Q}-4}\right. \\
& \left.\times \frac{\alpha_{Q}\left(\alpha_{Q}-1\right)\left(\alpha_{Q}-2\right)\left(\alpha_{Q}-3\right)}{2\left(2 \alpha_{Q}-1\right)}+\cdots\right\} .
\end{aligned}
$$

The expression for $\bar{f}_{11}{ }^{-\eta}$ is obtained from $\bar{f}_{11}{ }^{\eta}$ by interchanging $R$ and $Q$ in (2.14). Using Eqs. (2.13) and (2.14) as examples, we now examine the cases EE, EU, and UU individually.

\section{EE Scattering $\left(m_{a}=m_{c}=M, m_{b}=m_{d}=m\right)$}

From Eq. (2.3), $z=\nu / 4 p q$ where $\nu^{\alpha}$ is given by Eq. (2.7). $p q=\frac{1}{4}\left(t-4 M^{2}\right)^{1 / 2}\left(t-4 m^{2}\right)^{1 / 2}$ and $p q \rightarrow_{t \rightarrow 0}-M m$. In this case, for $s \rightarrow \infty,|z| \rightarrow \infty$ for all $t$, and there is no difficulty with the Regge expansion near $t=0$. Thus the first term in the expressions (2.13) and (2.14) is the leading term as $s \rightarrow \infty$ for any $t$, i.e.,

$$
\begin{array}{rll}
\bar{f}_{\mu \lambda} \eta & \sim(p q z)^{\alpha R-\Lambda} \rightarrow s^{\alpha R-\Lambda}, & \text { dominant parity } \\
& \sim(p q z)^{\alpha Q-\Lambda-1} \rightarrow s^{\alpha Q-\Lambda-1}, & \text { minor parity }
\end{array}
$$

and none of the succeeding terms for the $R$ or $Q$ trajectory contain singular coefficients. Therefore no daughters are required to ensure the analyticity of the PCHA, and we learn nothing about any possible family of poles associated with the trajectories $\alpha_{R}$ or $\alpha_{Q}$. Charge 
conjugation may restrict the $C$ of the exchanged poles, and hence may limit the number of members which could possibly contribute.

\section{EU Scattering}

Let $m_{a}=m_{c}=M, m_{b}=\mu, m_{d}=m$. Now we have

$$
p q=t^{-1 / 2}\left(t-4 M^{2}\right)^{1 / 2} T_{d b} / 4 \underset{t \rightarrow 0}{\rightarrow} \frac{-M\left|m^{2}-\mu^{2}\right|}{2 \sqrt{ }|t|} .
$$

$z=\nu / 4 p q$ and for $s \rightarrow \infty,|z|+\infty$ if $t$ is small enough. In fact, for $t=0, z_{t}=0$ for all $s$. This is the difficulty which historically was the motivation for the introduction of daughter trajectories. ${ }^{1}$

As an example, consider (2.13) for $\bar{f}_{10}{ }^{\eta}$. Using (2.5), (2.6), and (2.15), we see that the first term in the curly brackets of Eq. (2.13), which is proportional to $(p q z)^{\alpha-1}$, has a leading behavior $\sim s^{\alpha-1}$ followed by all succeeding lower powers; i.e.,

$$
(p q z)^{\alpha-1}=4^{1-\alpha} \nu^{\alpha-1} \rightarrow 2^{-\alpha} s^{\alpha-1}(1-\Sigma / 2 s)^{\alpha-1} .
$$

The second term in the curly brackets is proportional to

$$
(p q)^{2}(p q z)^{\alpha-3} \underset{t \rightarrow 0}{\stackrel{s \rightarrow \infty}{\longrightarrow}} \frac{-M^{2}\left(m^{2}-\mu^{2}\right)^{2}}{4 t} 2^{3-\alpha} S^{\alpha-3}(1-\Sigma / 2 s)^{\alpha-3} .
$$

Thus the factor $(p q)^{2}$ makes this term singular at $t=0$. Similarly, the third term is proportional to $(p q)^{4}$ $X(p q z)^{\alpha-5}$, which near $t=0$ is

and so on.

$$
\sim t^{-2} \nu^{\alpha-5}+O\left(t^{-1} \nu^{\alpha-5}\right)
$$

Note that besides the singular parts, each of the second and succeeding terms also contain pieces which are not singular (and do not vanish) at $t=0$; e.g.,

$$
\begin{gathered}
(p q)^{2}=-M^{2}\left(m^{2}-\mu^{2}\right)^{2} / 4 t+\frac{1}{16}\left[\left(m^{2}-\mu^{2}\right)^{2}\right. \\
\left.+8 M^{2}\left(m^{2}+\mu^{2}\right)\right]+O(t)+O\left(t^{2}\right), \\
(p q)^{4}=O\left(t^{-2}\right)+O\left(t^{-1}\right)+O\left(t^{0}\right)+\cdots .
\end{gathered}
$$

Analyticity requires that those terms more singular than the Wang $K(t)$ for the amplitude be removed from the PCHA, and this may be done for the EU case simply by including the contributions of the even daughters. The even daughters have the same $P$ and $C$ (and signature) as the parent pole and hence contribute to the same PCHA. In defining the formula for the contribution of the $n$th daughter (at $t=0$ ), we merely make the replacements $\gamma \rightarrow \gamma^{(n)}$ and $\alpha \rightarrow \alpha-n$ in all of the above expressions, i.e., at $t=0$,

$$
\bar{f}_{\mu 0^{\eta(n)}}=\bar{f}_{\mu 0}{ }^{\eta}\left(\gamma \rightarrow \gamma^{(n)}, \alpha \rightarrow \alpha-n\right) .
$$

The total contribution of the parent trajectory and all of its even daughters to the PCHA is then

$$
\bar{f}^{\eta}+\bar{f}^{\eta(2)}+\bar{f}^{\eta(4)}+\cdots \equiv \sum_{\text {even }} \bar{f}^{\eta(i)} .
$$

At this point it is interesting to note that the odd daughters will often not contribute to EU scattering because of $C .^{23}$ Thus it is particularly fortunate that their contributions are not required for singularity removal. (For a given family of poles, it may be that because of $C$ only the sequence of odd daughters contributes to a particular EU PCHA and performs the same cancellation which occurs in our discussion between the parent and even daughters.)

We determine the $t=0$ residues of the daughters $\gamma^{(n)}$ by requiring the sum in Eq. (2.20) to have no unwanted singularities. In this section, let us consider a simple case in which all singularities at $t=0$ allowed by the Wang kinematic factor occur in the leading term for the dominant amplitude $\left(\sim \nu^{\alpha-\Lambda}\right)$. In this case the singularity of the leading term is the over-all kinematic singularity of the amplitude, and the higher singularities which occur in the lower powers of $\nu$ must all be cancelled. More complicated cases are discussed in Secs. IV and V below, where the conspiracy relations at $t=0$ are taken into account.

To describe how daughter contributions remove undesired singularities, let us write schematically, at $t=0$,

$$
\begin{gathered}
\bar{f}_{\mu 0^{\eta}}=K \gamma\left[a_{0} \nu^{\alpha-\Lambda}+a_{2}(p q)^{2} \nu^{\alpha-\Lambda-2}\right. \\
\left.+a_{4}(p q)^{4} \nu^{\alpha-\Lambda-4}+\cdots\right], \\
\bar{f}_{\mu 0^{\eta(2)}}=K \gamma^{(2)}\left[a_{0(2) \nu^{\alpha-\Lambda-2}}+a_{2(2)}(p q)^{2} \nu^{\alpha-\Lambda-4}\right. \\
\left.+a_{4(2)}(p q)^{4} \nu^{\alpha-\Lambda-6}+\cdots\right], \\
\cdots \\
\bar{f}_{\mu 0^{\eta(n)}}=K \gamma^{(n)}\left[a_{0(n)} \nu^{\alpha-\Lambda-n}\right. \\
\left.+a_{2(n)}(p q)^{2} \nu^{\alpha-\Lambda-2-n}+\cdots\right], \\
\cdots
\end{gathered}
$$

where

$$
a_{l(n)}(\alpha)=a_{l}(\alpha-n) .
$$

As we have seen, singularities arise in these expansions because $(p q)^{2} \sim 1 / t$. To remove singularities in the sum, $\sum_{\text {even }} \bar{f}^{\eta}$, we require

$$
\begin{array}{r}
{\left[t\left[\gamma a_{2}(p q)^{2}+\gamma^{(2)} a_{0(2)}\right]\right]_{t=0}=[0(t)]_{0}=0, \quad(2.22 \mathrm{a})} \\
\left\{t^{N}\left[\gamma a_{4}(p q)^{4}+\gamma^{(2)} a_{2(2)}(p q)^{2}+\gamma^{(4)} a_{0(4)}\right]\right\}_{t=0} \\
=\left[O\left(t^{N}\right)\right]_{0}=0, \text { for } N=2,1, \quad(2.22 \mathrm{~b}) \\
\cdots \\
\text { for } N=k, k-1, \cdots, 1 . \quad(2.22 \mathrm{k})
\end{array}
$$

Equation (2.22a) implies that at $t=0, \gamma^{(2)} / \gamma=C_{1}{ }^{(2)} / t$ $+C_{0}{ }^{(2)}$, where the $1 / t$ part (i.e., $C_{1}$ ) is determined by

$$
\left[t \gamma^{(2)} / \gamma\right]_{t=0}=-\left[a_{2} t(p q)^{2} / a_{0(2)}\right]_{t=0}=C_{1}{ }^{(2)} .
$$

${ }^{23}$ In particular, if particles $a$ and $c$ are identical fermions, then when the helicities of the $t$-channel equal-mass pair are $\lambda_{3}= \pm \lambda_{4}$, we are restricted to the exchange of a single particular $C= \pm(-1)^{j}$ for each parity part of the PCHA. For the $N \bar{N}$ system, we always have $\lambda_{3}= \pm \lambda_{4}$. When $\lambda_{3}=\lambda_{4}$, we have $\mu=0$ and the sole term has $C=+(-1)^{J}$. For $\lambda_{3}=-\lambda_{4}$, the minor parity term has opposite $P$ and $C$ from the dominant term, where both terms have $C P$ $=+1$. So either the first has $P=C=+(-1)^{J}$ and the other $P=C=-(-1)^{J}$ or vice versa. 
Using this result, Eq. (2.22b) implies (at $t=0$ )

$$
\gamma^{(4)} / \gamma=C_{2}{ }^{(4)} / t^{2}+C_{1}{ }^{(4)} / t+C_{0}{ }^{(4)}
$$

where (2.22b) with $N=2$ determines $C_{2}{ }^{(4)}$ once $C_{1}{ }^{(2)}$ is known, and (2.22b) with $N=1$ determines $C_{1}{ }^{(4)}$ once $C_{0}{ }^{(2)}$ has been specified. Note that the less singular parts of a residue are not determined by singularity cancellation unless the constant pieces of higher daughter residues are specified.

It is obvious from the system of equations that we obtain, in general, for the singular pieces of the even residues (in EU scattering)

$\gamma^{(2 l)} / \gamma=\sum_{n=1}^{l} C_{n}(2 l) / t^{n}, \quad$ where $C_{n} \propto\left(m^{2}-\mu^{2}\right)^{2 n}$.

Thus for the most singular piece

$$
\gamma^{(2 l)} / \gamma \sim\left(m^{2}-\mu^{2}\right)^{2 l} / t^{l} .
$$

from (2.25) we see that if the parent residue $\gamma$ is nonsingular, all of its even daughters have singular residues.

If the parent residue $\gamma$ has a zero of order $t^{n}$, the residues of the first $n$ (even) daughters $\left(\gamma^{(2)}, \gamma^{(4)}, \cdots\right.$, $\left.\gamma^{(2 n)}\right)$ are not fixed by analyticity. The most singular behavior of the remaining daughters is, however, fixed by specifying the behavior of these first $n$. This freedom in daughter residues in the event of evading parent trajectories is discussed for a special case in Sec. V.

Note, from Eq. (2.25), that the singular pieces of the daughter residues vanish for $m=\mu$, i.e., the coupling is nonsingular for an equal-mass vertex. This agrees with what we found above for the $\mathrm{EE}$ case, where no daughters were required. Adding up the contributions [Eq. (2.21)] of all the even daughters, we have

$$
\begin{aligned}
& \sum_{\text {even }} \bar{f}_{\mu 0^{\eta}}= K\left\{\left[\gamma a_{0}\right] \nu^{\alpha-\Lambda}+\left[\gamma a_{2}(p q)^{2}+\gamma^{(2)} a_{0(2)}\right] \nu^{\alpha-\Lambda-2}\right. \\
&+\cdots+\left[\sum_{l=0,2, \ldots}^{2 k} \gamma^{(l)} a_{2 k-l(l)}(p q)^{2 k-l}\right] \\
&\left.\times \nu^{\alpha-\Lambda-2 k}+\cdots\right\},
\end{aligned}
$$

where the singular $(t=0)$ terms in each square bracket [ ] have been removed by the constraints $(2.22 \mathrm{a}, \mathrm{b}, \cdots)$. Note that if $\alpha$ is not an integer, our sum includes an infinite series of daughter trajectories.

Then, with daughters included, the first term in (2.27) dominates at large $s$ for all $t$ and we have (for any $\Lambda$ )

$$
\sum_{\text {even }} \bar{f}_{\Lambda 0^{\eta}} \underset{s \rightarrow \infty}{\rightarrow} s^{\alpha-\Lambda} .
$$

Hence, daughters make the leading term in the Regge expansion of the unequal-mass PCHA dominate and look identical to the leading term in an expansion of equal-mass scattering. This property has been used by many authors ${ }^{7,15,17,24,25}$ in previous Regge analyses.

\footnotetext{
${ }^{24}$ H. Shepard, Phys. Rev. 159, 1331 (1967).

25 The number of actual fits made using this result is far too large for enumeration.
}

Note that a parity-doubled family regularizes $\bar{f}_{\mu 0}$ in the same way as above, since only one of the parity families contributes to this PCHA.

We may in a similar manner consider the EU case when $\lambda$ and $\mu$ are both nonzero. Now we have, from (2.11) and (2.12),

$$
\begin{gathered}
\bar{f}_{\mu \lambda} \eta K^{R} \gamma^{R} \chi^{R}\left[a_{0}{ }^{R} \nu^{\alpha R-\Lambda}+a_{2}{ }^{R}(p q)^{2} \nu^{\alpha R^{-\Lambda-2}}+\cdots\right] \\
+K^{Q} \gamma^{Q} \chi^{Q}(p q)\left[a_{1} \nu^{\alpha Q^{-\Lambda-1}}\right. \\
\left.+a_{3}{ }^{Q}(p q)^{2} \nu^{\alpha^{Q}-\Lambda-3}+\cdots\right]
\end{gathered}
$$

From our previous discussion it is obvious that the singular terms in the square bracket [ $]$ of the dominant-parity part can be removed by including the even daughters of $\alpha_{R}$ with suitably chosen residues, and similarly the even daughters of $\alpha_{Q}$ cancel the unwanted $t=0$ singularities of the minor-parity part of $\bar{f}_{\mu \lambda}{ }^{\eta}$. (See Sec. IV for further comments.) It can be demonstrated that the same set of daughter residues which regularizes $\bar{f}_{\mu \lambda}{ }^{\eta}$ also corrects the analytic structure of $\bar{f}_{\mu \lambda}{ }^{-\eta}$, as it must. A proof is given for the case $\lambda=\mu=1$ in Appendix B.

It is worthwhile to notice at this point that one does not have to consider terms in the daughter residue function $\gamma^{(2 n)}$ less singular than $1 / t^{n}$, in order to do proofs about singularity cancellation or relations to other amplitudes. Provided proofs can be carried through for the most singular term, they follow automatically for less singular terms; the arguments for less singular terms are analogous to those for a parent residue with the canonical residue function multiplied by powers of $t$.

To summarize: We have demonstrated that the proper analytic properties of the individual PCHA for EU scattering can be achieved by including the contribution from the infinite sum of even daughters of a (single) parent pole. No odd daughters were required. We have shown that these (even) daughters must have singular residues at $t=0$ as given in Eqs. (2.25) and (2.26). With the daughter contributions added, the resulting PCHA is dominated at large $s$ by the leading term in the dominant-parity or minor-parity part of the amplitude, viz., $\bar{f}_{\mu \lambda^{\eta}} \sim s^{\alpha_{R}-\Lambda}$ or $\sim s^{\alpha Q-\Lambda-1}$, whichever is larger.

We can briefly note how a different pattern of singularity cancellation by daughters occurs for the parity-doubled (EU) case. An example (in $\pi N \rightarrow V N$ ) will be given in Sec. V.

Consider Eq. (2.28) and assume that the dominantand minor-parity parts of the PCHA also have definite $C$. If $(p q) K^{Q}$ is more singular than the maximum singularity allowed for this amplitude (viz., $K^{R}$ ), the even daughters of two single-parity families $\alpha_{R}$ and $\alpha_{Q}$ cancel all singularities except that in the $\left(p q K^{Q}\right)$ part of the coefficient of minor parity terms. We therefore require an extra (evasive) factor of $t$ in $\gamma^{Q}$ in order that Regge pole $Q$ and its daughters have a properly behaved contribution.

But we may avoid these evasive zeros with a paritydoubled family of poles (where the parents have the 
same $C$ ). We would need $\alpha_{R}=\alpha_{Q} \pm 1$ and either $Q$ is the odd daughter of the leading partner of $R$, or $R$ is the odd daughter of the partner of $Q$. Then only the odd daughters of one family contribute to the minor-parity part of the PCHA, and the parent and even daughters of the other family contribute to the dominant-parity part, or vice versa.

For example, with $\alpha=\alpha_{Q}=\alpha_{R}+1$,

$$
\bar{f}_{\mu \lambda} \rightarrow\left[K^{R} \gamma^{R} a_{0}+(p q) K^{Q} \gamma^{Q} a_{1}\right] \nu^{\alpha-\Lambda-1}+\cdots,
$$

and a cancellation occurs so that the square bracket has a singularity no worse than $K^{R}$. In the example in Sec. $\mathrm{V}$, this requires $\gamma^{R}$ (which is the residue of the first daughter of the parity-doubled partner of $Q$ ) to have a singular part. Thus, in contrast to the single-parity case, both odd and even daughters (of different parents, however) contribute in the EU case and cancel singularities arising from members of both parity families. In the example begun above, one can show that $\bar{f}_{\mu \lambda} \rightarrow \eta$ has an expansion with leading term $\nu^{\alpha-\Lambda}$ and all succeeding even powers of $\nu$. The daughters required for the regularization of $\bar{f}^{\eta}$ and $\bar{f}^{-\eta}$ have maximum singularities $\gamma^{(1)}$ and $\gamma^{(2)} \sim t^{-1}, \gamma^{(3)}$ and $\gamma^{(4)} \sim t^{-2}$, etc., where it must be remembered that the even and odd daughters have opposite-parity parents. This case can be worked in detail employing the procedure already described.

\section{UU Scattering $\left(m_{a} \neq m_{c}\right.$ and $\left.m_{b} \neq m_{d}\right)$}

From Eqs. (2.2), (2.3), and (2.6) we have

$$
\begin{gathered}
(p q)=T_{a c} T_{d b} / 4 t \underset{t \rightarrow 0}{\rightarrow}\left|\Delta_{a c} \Delta_{b d}\right| / 4 t \equiv\left|\Delta_{m}\right| / 4 t, \quad z=\nu^{\prime} / 4 p q=\left(\nu+\Delta_{m} / t\right)(4 p q)^{-1}, \\
(z \mp 1) \underset{t \rightarrow 0}{\rightarrow} \pm(4 p q)^{-1}\left[\nu+\Delta_{b d} \Delta_{a c}{ }^{-1}\left(m_{a}{ }^{2}+m_{c}^{2}\right)+\Delta_{a c} \Delta_{b d}{ }^{-1}\left(m_{b}{ }^{2}+m_{d}{ }^{2}\right)\right] \text { for } \quad \Delta_{a c} \Delta_{b d} \gtrless 0 .
\end{gathered}
$$

Thus, as in the EU case, $|z|+\rightarrow \infty$ for $s \rightarrow \infty$ if $t$ is small (for $t=0, z_{t}= \pm 1$ for all $s$ for $\Delta_{d b} \Delta_{c a} \gtrless 0$ ). Because neither $t$-channel state is a particle-antiparticle pair, the charge conjugation of the exchanged state is in general unrestricted.

For UU scattering it is $\bar{f}_{34,12}$ rather than the PCHA which has an easily specified analytic behavior at $t$ $=0{ }^{7,26}$ The maximum singularity (for $\Delta_{a c} \Delta_{b d}>0$ ) allowed by the requirement that $f_{34,12}$ be nonsingular is $\bar{f}_{34,12} \sim(\sqrt{ } t)^{-|\lambda-\mu|}$. The conspiracy relations for PCHA follow directly from this. (See Sec. III.)

For $\lambda=0$, since $K_{\mu 0}=K_{-\mu 0}$, we can expand the PCHA

$$
\begin{aligned}
\bar{f}_{\mu 0}{ }^{\eta} \rightarrow \sum_{i=1,2}\left(2 \alpha_{i}+1\right) T_{\mu 0}{ }^{\alpha_{i}} e_{0 \mu}{ }^{\alpha_{i}{ }^{+}} \\
\rightarrow K_{\mu 0} \sum_{i} \gamma_{\mu 0} \chi \chi\left(\tau_{i}\right)\left[a_{0}{ }^{i}\left(\nu^{\prime}\right)^{\alpha_{i}-\Lambda}+a_{2}{ }^{i}(p q)^{2}\left(\nu^{\prime}\right)^{\alpha_{i}-\Lambda-2}\right. \\
\left.+a_{4}{ }^{i}(p q)^{4}\left(\nu^{\prime}\right)^{\alpha_{i}-\Lambda-4}+\cdots\right], \quad(2.30)
\end{aligned}
$$

where the poles $\alpha_{1}(i=1)$ and $\alpha_{2}(i=2)$ have the same parity but opposite charge conjugation. The expansion of $\nu^{\prime}$, Eq. (2.6), shows that even for a single pole the odd powers of $\nu$ (modulo $\left.\nu^{\alpha-\Lambda}\right)$, as well as the even powers, are present, and all have coefficients which are singular at $t=0$. Thus, for UU scattering the situation is more complicated than the corresponding EU case.

Expanding, using (2.6), we have

$$
\begin{array}{r}
\bar{f}_{\mu 0} \eta \sum_{i} K \chi_{\gamma}\left\{a_{0}\left[\sum_{l=0}^{\alpha-\Lambda}\left(\begin{array}{c}
\alpha-\Lambda \\
l
\end{array}\right)\left(\frac{\Delta_{m}}{t}\right)^{l} \nu^{\alpha-\Lambda-l}\right]+a_{2}(p q)^{2}\left[\sum_{l=0}^{\alpha-\Lambda-2}\left(\begin{array}{c}
\alpha-\Lambda-2 \\
l
\end{array}\right)\left(\frac{\Delta_{m}}{t}\right)^{l} \nu^{\alpha-\Lambda-2-l}\right]+\cdots\right. \\
\left.+a_{2 k}(p q)^{2 k}\left[\sum_{l=0}^{\alpha-\Lambda-2 k}\left(\begin{array}{c}
\alpha-\Lambda-2 k \\
l
\end{array}\right)\left(\frac{\Delta_{m}}{t}\right)^{l} \nu^{\alpha-\Lambda-2 k-l}\right]+\cdots\right\}=\sum_{i} K \chi_{\gamma}\left\{\nu^{\alpha-\Lambda}\left[\left(\begin{array}{c}
\alpha-\Lambda \\
0
\end{array}\right) a_{0}\right]+\nu^{\alpha-\Lambda-1}\left[\left(\begin{array}{c}
\alpha-\Lambda \\
1
\end{array}\right)\left(\frac{\Delta_{m}}{t}\right) a_{0}\right]\right. \\
\left.+\nu^{\alpha-\Lambda-2}\left[\left(\begin{array}{c}
\alpha-\Lambda \\
2
\end{array}\right)\left(\frac{\Delta_{m}}{t}\right)^{2} a_{0}+\left(\begin{array}{c}
\alpha-\Lambda-2 \\
0
\end{array}\right)(p q)^{2} a_{2}\right]+\cdots\right\} .
\end{array}
$$

For $m_{a}=m_{c}$, the odd powers $\left(\nu^{\alpha-\Lambda-1}, \nu^{\alpha-\Lambda-3}, \cdots\right)$ vanish, and (2.31) reduces to the EU case. But for UU all powers of $\nu$ are present, and since $(p q)^{2} \sim 1 / t^{2}$, the coefficient of $\nu^{\alpha-\Lambda-n}$ has maximum singularity $t^{-n}$. (For EU it is $t^{-n / 2}$ with only even $n$ appearing.)

Consider the contribution of a single parent pole and all of its daughters to $\vec{f}_{\mu 0}{ }^{\eta}$. In contrast to the EU case, the odd daughters (which have opposite $C$ from the leading pole and the even daughters) always can contribute to the same UU PCHA as the parent. This is

${ }^{26}$ J. D. Jackson and G. E. Hite, Phys. Rev. 169, 1248 (1968). fortunate since the odd daughters are necessary if we are to cancel singularities in all of the coefficients in (2.31).

We represent the contribution of the $n$th daughter at $t=0$ by

$$
\bar{f}_{\mu 0^{\eta(n)}}=\bar{f}_{\mu 0^{\eta}}\left(\gamma \rightarrow \gamma^{(n)}, \alpha \rightarrow \alpha-n\right) .
$$

Notice that at $t=0$ the signature-pole factor $\chi$ is the same for the parent and all of its daughters, since with $\tau_{(n)}=(-1)^{n} \tau$, we have

$\left[e^{-i \pi(\alpha-n)}+\tau_{(n)}\right][\sin \pi(\alpha-n)]^{-1}=\left[e^{-i \pi \alpha}+\tau\right][\sin \pi \alpha]^{-1}$. 
In order that the sum of parent and daughter contributions $\sum \bar{f}_{\mu 0^{\eta}}$ be free of unwanted singularities, we require

$$
\begin{aligned}
& \left\{t\left[\left(\begin{array}{c}
\alpha-\Lambda \\
1
\end{array}\right) \frac{\Delta_{m}}{t} \gamma a_{0}+\left(\begin{array}{c}
\alpha-\Lambda-1 \\
0
\end{array}\right) \gamma^{(1)} a_{0(1)}\right]\right\}_{t=0}=[O(t)]_{0}=0
\end{aligned}
$$

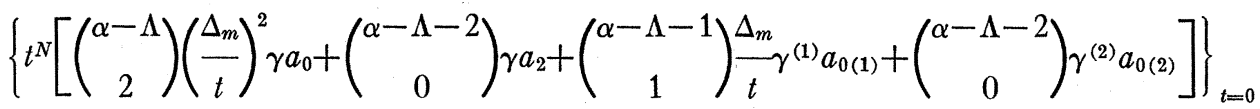

$$
\begin{aligned}
& =\left[O\left(t^{N}\right)\right]_{0}=0 \text { for } N=2,1, \\
& \left.\left[t^{N} \text { (coefficient of } \nu^{\alpha-\Lambda-n} \text { in } \sum \tilde{f}_{\mu 0^{\eta}}\right)\right]_{t=0}=\left[O\left(t^{N}\right)\right]_{0}=0 \text { for } N=n, n-1, \cdots, 1 \text {. }
\end{aligned}
$$

Equations (2.32) have as their solution daughter residues with singular pieces

$$
\gamma^{(n)} / \gamma=C_{n} / t^{n}+C_{n-1} / t^{n-1}+\cdots+C_{1} / t
$$

where

$$
C_{n} \sim\left(\Delta_{a c} \Delta_{b d}\right)^{n} .
$$

Thus, if the parent residue $\gamma$ is nonsingular, all of its daughters have singular residues. ${ }^{27}$ As in the EU case, evasive behavior (for $\gamma$ ) makes some daughter residues nonsingular and hence unrestricted by the analyticity requirements (singularity cancellation). This freedom becomes important when factorization is considered (Sec. IV).

With all unwanted singularities removed according to Eq. (2.32), the dominant term in $\sum \bar{f}_{\mu 0}{ }^{0}$ for large $s$ (any $t$ ) is the first term

$$
\sum \bar{f}_{\mu 0^{\eta}} \rightarrow K \chi \gamma a_{0} \nu^{\alpha-\Lambda} \sim s^{\alpha-\Lambda},
$$

and thus for UU scattering (with $\lambda=0$ ), daughters preserve the simple (equal-mass) Regge asymptotic formula. Because only one parity contributes to $\bar{f}_{\mu 0}$, the result is the same for a parity-doubled family of poles.

With $\lambda$ and $\mu$ nonzero, we noted above that $\bar{f}_{\mu \lambda}$ and $\bar{f}_{-\mu \lambda}$ are allowed different $t=0$ singularities. Thus, in general, the PCHA $\bar{f}^{\eta}$ and $\bar{f}^{-\eta}$ may both have maximum singularity

where

$$
\left(\bar{f}_{\mu \lambda} \pm \bar{f}_{-\mu \lambda}\right) \sim(\sqrt{ } t)^{-\xi}
$$

$$
\xi=\max (|\lambda+\mu|,|\lambda-\mu|) \text {. }
$$

However, for a single parity exchange, it is easy to see that the leading term $\left(\sim \nu^{\alpha-\Lambda}\right)$ in $\bar{f}_{\mu \lambda}$ and $\bar{f}_{-\mu \lambda}$ must be the same (up to a sign). This follows from

$$
\left(\bar{f}_{ \pm \mu \lambda}\right) \rightarrow(2 \alpha+1) T_{\mu \lambda} \lambda^{\eta \alpha}\left(e_{\lambda \mu}{ }^{\alpha+} \pm e_{\lambda \mu}{ }^{\alpha-}\right)
$$

(modulo an over-all sign depending on the parity of the exchanged pole) and we observe that the $\nu^{\alpha-\Lambda}$ term which comes from $e^{\alpha+}$ is common to both $\bar{f}_{ \pm \mu \lambda}$. Hence, $\bar{f}_{\mu \lambda}$ and $\bar{f}_{-\mu \lambda}$ have a common over-all singularity multiplying their highest power of $\nu$. This can be no worse

27 Equation (2.33) has been written in a manner which does not make evident the change of behavior as $\Delta_{a c} \rightarrow 0$ in the transition from the UU to the EU case. All the formulas for the UU case do go over smoothly to the formulas for the EU case, but this is most easily seen if appropriate terms are written in terms of $(p q)^{n}$ rather than $\left(\Delta_{a c} \Delta_{b d} / 4 t\right)^{n}$. than the minimum common singularity, viz., $(\sqrt{ } t)^{-\delta}$ where $\delta=\min (|\lambda+\mu|,|\lambda-\mu|)$. Thus for single-parity exchange, choosing $|\lambda+\mu|>|\lambda-\mu|$ to be specific, if $\bar{f}_{\mu \lambda}$ has its maximum allowable singularity removed as an over-all factor $K_{\mu \lambda}$, we then permit no further singularities to occur in coefficients of nonleading terms when daughters have been included with suitably chosen residues. This same single-parity family also contributes to $\bar{f}_{-\mu \lambda}$. We have

$$
\begin{aligned}
& \bar{f}_{\mu \lambda} \rightarrow \gamma K_{\mu \lambda}(p q)^{\alpha-\Lambda \frac{1}{2}\left(e_{\mu \lambda}{ }^{\alpha+}+e_{\lambda \mu}{ }^{\alpha-}\right),}
\end{aligned}
$$

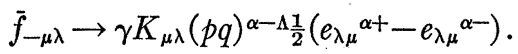

Because we have removed from $\bar{f}_{-\mu \lambda}$ a factor $K_{\mu \lambda}$ and not $K_{-\mu \lambda}$, terms as singular as $(\sqrt{ } t)^{-|\lambda+\mu|+|\lambda-\mu|}$ are allowed to occur in nonleading powers of $\bar{f}_{-\mu \lambda}$ even when the daughter contributions have been included. The way in which this happens is demonstrated (for the case $\lambda=\mu=1$ ) in Appendix C. (See also some further comments in Sec. IV.) With the even and odd daughters of a single-parity family included, we therefore (for the case $\lambda$ and $\mu$ nonzero) are again able to remove all unwanted singularities.

In order that both $\bar{f}_{\mu \lambda}$ and $\bar{f}_{-\mu \lambda}$ have their maximum allowed kinematic singularity multiplying the leading power, we must consider the contribution of a paritydoubled family. Schematically, if the trajectories $\alpha_{x}$ and $\alpha_{y}$ have opposite parities, they contribute to $\bar{f}_{ \pm \mu \lambda}$ as follows:

$$
\begin{gathered}
\bar{f}_{\mu \lambda} \sim a\left(\nu^{\alpha_{x}-\Lambda}+\cdots\right)+A\left(\nu^{\alpha_{y}-\Lambda}+\cdots\right), \\
\bar{f}_{-\mu \lambda} \sim a\left(\nu^{\alpha_{x}-\Lambda}+\cdots\right)-A\left(\nu^{\alpha_{y}-\Lambda}+\cdots\right) .
\end{gathered}
$$

With $\alpha_{x}=\alpha_{y}=\alpha$,

$$
\begin{aligned}
\bar{f}_{\mu \lambda} & \sim(a+A) \nu^{\alpha-\Lambda}, \\
\bar{f}_{-\mu \lambda} & \sim(a-A) \nu^{\alpha-\Lambda},
\end{aligned}
$$

and we are now allowed to have $K_{\mu \lambda} \sim(a+A)$ $\sim(\sqrt{ } t)^{-|\lambda-\mu|}$ and $K_{-\mu \lambda} \sim(a-A) \sim(\sqrt{ } t)^{-|\lambda+\mu|}$, and the $\tilde{f}_{ \pm \mu \lambda}$ no longer have a common over-all singularity.

Since we now can factor out separately the maximum allowed singularity from $\bar{f}_{\mu \lambda}$ and $\bar{f}_{-\mu \lambda}$, the subsidiary terms of each are allowed no further singularities. Because of this, the pattern of singularity cancellation by daughters in $\sum \bar{f}_{\mu \lambda}$ and $\sum \bar{f}_{-\mu \lambda}$ is different from the case of a single-parity family. 
TABLE I. Amplitudes for $V \pi \rightarrow N \bar{N}, V \pi \rightarrow V \pi$, and $\bar{N} N \rightarrow N \bar{N}$.

\begin{tabular}{|c|c|c|}
\hline Amplitude & $K(t)$ & Dominant $P, C$ \\
\hline 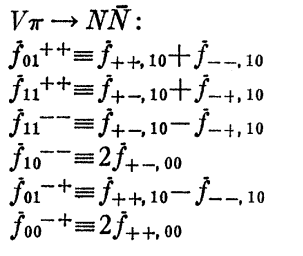 & $\begin{array}{c}1 \\
1 / \sqrt{ } t \\
1 \\
1 / \sqrt{ } t \\
1 / \sqrt{ } t \\
1 / \sqrt{ } t\end{array}$ & $\begin{array}{l}P=C=+(-1)^{J} \\
P=C=+(-1)^{J} \\
P=C=-(-1)^{J} \\
P=C=-(-1)^{J} \\
P=-C=-(-1)^{J} \\
P=-C=-(-1)^{J}\end{array}$ \\
\hline $\begin{array}{l}V \pi \rightarrow V \pi: \\
\tilde{f}_{11}{ }^{+} \equiv \tilde{f}_{10,10}-\tilde{f}_{-10,10} \\
\tilde{f}_{11}=\dot{f}_{10,10}+\bar{f}_{-10,10} \\
\tilde{f}_{00} \equiv 2 \bar{f}_{00,00} \\
\tilde{f}_{10}{ }^{-} \equiv 2 \bar{f}_{10,00}\end{array}$ & $\begin{array}{c}1 / t \\
1 / t \\
1 \\
1 / \sqrt{ } t\end{array}$ & $\begin{array}{l}P=(-1)^{J}, C= \pm(-1)^{J} \\
P=-(-1)^{J}, C= \pm(-1)^{J} \\
P=-(-1)^{J}, C= \pm(-1)^{J} \\
P=-(-1)^{J}, C= \pm(-1)^{J}\end{array}$ \\
\hline $\begin{aligned} \bar{N} N & \rightarrow N \bar{N}: \\
f_{2} & \equiv \tilde{f}_{++,++}+\bar{f}_{--,++} \\
f_{4} & \equiv \bar{f}_{+-,++}+\tilde{f}_{-+,+-} \\
f_{5} & \equiv \tilde{f}_{++,+-}+\bar{f}_{--,+-} \\
f_{3} & \equiv \tilde{f}_{+-,+-}-\bar{f}_{-+,+-} \\
f_{1} & \equiv \tilde{f}_{++,++}-\tilde{f}_{--,++}\end{aligned}$ & $\begin{array}{l}1 \\
1 \\
\sqrt{ } t \\
1 \\
1\end{array}$ & $\begin{array}{l}P=C=+(-1)^{J} \\
P=C=+(-1)^{J} \\
P=C=+(-1)^{J} \\
P=C=-(-1)^{J} \\
P=-C=-(-1)^{J}\end{array}$ \\
\hline
\end{tabular}

For a double-parity family, we find

$$
\begin{gathered}
\sum \bar{f}_{ \pm \mu \lambda} \sim K_{ \pm \mu \lambda}\left\{\nu^{\alpha-\Lambda}\left[\left(\begin{array}{c}
\alpha-\Lambda \\
0
\end{array}\right)\left(\gamma_{u} a_{0}{ }^{u} \pm \gamma_{v} a_{0}{ }^{v}\right)\right]\right. \\
+\nu^{\alpha-\Lambda-1}\left[\left(\begin{array}{c}
\alpha-\Lambda \\
1
\end{array}\right)\left(\Delta_{m} / t\right)\left(\gamma_{u} a_{0}{ }^{u} \pm \gamma_{v} a_{0}{ }^{v}\right)\right. \\
\pm p q\left(\gamma_{u} a_{1}{ }^{u} \pm \gamma_{v} a_{1}{ }^{v}\right)+\left(\gamma_{u}{ }^{(1)} a_{0(1)}{ }^{u}\right. \\
\left.\left.\left. \pm \gamma_{v}{ }^{(1)} a_{0(1)}{ }^{v}\right)\right]+\cdots\right\},
\end{gathered}
$$

where $u$ and $v$ refer to the opposite-parity families $\left(\alpha_{u}=\alpha_{v}=\alpha\right)$. Because the coefficients $a_{k}$ depend only on the $\alpha$ of the trajectory involved, $a_{k}{ }^{u}=a_{k}{ }^{v}$ at $t=0$. Thus, Eq. (2.37) may be rewritten in terms of $\gamma_{u}+\gamma_{v}$ and $\gamma_{u}-\gamma_{v}$. To remove the $1 / t$ singularity in the coefficient of $\nu^{\alpha-\Lambda-1}$ for $\sum \bar{f}_{\mu \lambda}$ and $\sum \bar{f}_{-\mu \lambda}$, we require

$$
\begin{array}{r}
\left\{t\left[\text { coefficient of } \nu^{\alpha-\Lambda-1} \text { in Eq. }(2.37)\right]\right\}_{t=0} \\
=[O(t)]_{0}=0 .
\end{array}
$$

Equation (2.38) is actually two equations, one for the upper signs in (2.37) and one for the lower signs. Adding and subtracting these two equations, we have

$$
\begin{aligned}
& \left(\begin{array}{c}
\alpha-\Lambda \\
1
\end{array}\right) \Delta_{m} \gamma_{u} a_{0}^{u}+(p q t) \gamma_{v} a_{1}^{v}=-t \gamma_{u}^{(1)} a_{0(1)}{ }^{u}, \\
& \left(\begin{array}{c}
\alpha-\Lambda \\
1
\end{array}\right) \Delta_{m} \gamma_{v} a_{0}{ }^{v}+(p q t) \gamma_{u} a_{1}^{u}=-t \gamma_{v}^{(1)} a_{0(1)}{ }^{v} .
\end{aligned}
$$

This illustrates that we cannot decouple the $u$ and $v$ families. The daughters in the $u$ family cancel singularities arising from higher poles in both the $u$ and $v$ families and similarly for $v$-family daughters. Although we shall not pursue it further here, by using the singularity-cancellation procedure one can calculate the singular parts of the lower daughter residues as well. In general (for nonevasive parent residues), one finds maximum singularities $\gamma^{(1)} \sim t^{-1}, \gamma^{(2)} \sim t^{-2}, \cdots, \gamma^{(n)} \sim t^{-n}$ where the behavior indicated holds for each of the two daughter residues, $\gamma_{u}{ }^{(n)}$ and $\gamma_{v}{ }^{(n)}$.

The UU parity-doubled case is illustrated in Sec. V for $\pi \pi \rightarrow V V$ scattering.

\section{CONSPIRACY}

The conspiracy relations are restrictions on the analytic behavior of linear combinations of helicity amplitudes at $t=0$. These kinematic constraints are necessary to complete the description of the analytic properties of the amplitudes and must be considered in any attempt to find the restrictions imposed by analyticity on patterns of pole structure.

For the reactions considered as examples below $(\pi \pi \rightarrow V V, N N \rightarrow N N, \pi N \rightarrow V N)$, the conspiracy relations are easily obtained and have been cited several times in the literature. We list them here for the sake of completeness and for use in later sections. The amplitudes given below are defined in Table $\mathrm{I}$.

(a) In nucleon-nucleon scattering, the relation originally found by Volkov and Gribov ${ }^{8}$

$$
f_{++,++}{ }^{t}-f_{++,--}{ }^{t}=f_{+-,+-}{ }^{t}-f_{+-,-+^{t}}
$$

may be written in the form

$$
\begin{aligned}
& \bar{f}_{++,++}-\bar{f}_{++,--}=\left(\bar{f}_{+-,+-}-\bar{f}_{+-,-+}\right) \\
& +z_{t}\left(\bar{f}_{+-,+-}+\bar{f}_{+-,-+}\right)
\end{aligned}
$$

or

$$
f_{1}=f_{3}+z_{l} f_{4} \quad(\text { at } t=0),
$$

which shows more clearly the role of contributions from different PCHA (hence different quantum numbers). The $O(3,1)$ interpretation of this equation has been given by Freedman and Wang. ${ }^{4}$

(b) The general conspiracy relations for processes of the type $1+N \rightarrow 2+N$ have been shown by $\mathrm{Stack}^{28}$ to take the form (at $t=0$ )

$$
i\left[\bar{f}_{++, 12}-\bar{f}_{--, 12}\right]+\left[\bar{f}_{+-, 12}+\bar{f}_{-+, 12}\right]=0 .
$$

For the process $\pi N \rightarrow V N$, this gives two relations ${ }^{16,29}$ one involving vector-meson helicity 1 and one involving vector-meson helicity 0 .

$$
\begin{aligned}
& {\left[(\sqrt{ } t) \bar{f}_{10}^{--}\right]_{t=0}=-i\left[(\sqrt{ } t) \bar{f}_{00}{ }^{+}\right]_{t=0},} \\
& {\left[(\sqrt{ } t) \bar{f}_{11}{ }^{++}\right]_{t=0}=-i\left[(\sqrt{ } t) \bar{f}_{01}{ }^{-+}\right]_{t=0} .}
\end{aligned}
$$

(c) For $t$-channel processes of the UU form, there are no constraints at $t=0$ for the separate helicity amplitudes, $\bar{f}_{34,12}$, but there are relations between the PCHA which must be satisfied to ensure proper singularity

${ }^{28}$ J. Stack, Phys. Rev. 171, 1666 (1968).

${ }^{29} \mathrm{H}$. Högaasen and Ph. Salin, Nucl. Phys. B2, 657 (1967). 
TABLE II. Residues of parent- and first-daughter trajectories in a family with single leading $P=C=(-1)^{J}$ pole. The $t=0$ residues given [defined in Eqs. (2.8) and (2.10)] are consistent with factorization, analyticity, and the conspiracy relations. Each residue in the table has been divided by $(2 \alpha+1)$, where $\alpha$ is the $t=0$ intercept of the leading trajectory. Only the most singular term in the daughter residue is to be evaluated from the given entry. The momenta are $p=\frac{1}{2}\left(t-4 M^{2}\right)^{1 / 2}, q=T_{\pi V} / 2 t^{1 / 2}$, and $\Delta=\left(m V^{2}-m_{\pi}^{2}\right)$. The functions $B_{i}$ are analytic at $t=0$, but not necessarily at other values of $t$. The energy dependence of each amplitude, shown in the column to the left of the residue, comes from the first term in the expansion of the $e$ function, which multiplies this residue. ${ }^{\mathrm{a}}$

\begin{tabular}{|c|c|c|}
\hline Amplitude & Residue of leading trajectory & Residue of first-daughter trajectory \\
\hline \multicolumn{3}{|l|}{$\pi N \rightarrow V N:$} \\
\hline$\vec{f}_{01}^{++}$ & $(s / p q)^{\alpha-1} B_{u}^{*} B_{w}(p q)^{\alpha-1} p$ & \\
\hline $\bar{f}_{11}++$ & $(s / p q)^{\alpha-1}(1 / \sqrt{ } t) t B_{v}^{*} B_{w}(p q)^{\alpha-1}$ & \\
\hline$\tilde{f}_{11}^{--}$ & $(s / p q)^{\alpha-2}(1 / \sqrt{ } t) t B_{v}{ }^{*} B_{w}(p q)^{\alpha-1}$ & \\
\hline \multicolumn{3}{|l|}{$\pi \pi \rightarrow V V:$} \\
\hline \multirow{2}{*}{\multicolumn{3}{|c|}{$\left(s / q^{2}\right)^{\alpha-1}(1 / t) t B_{w}{ }^{*} B_{w}\left(q^{2}\right)^{\alpha-1}$}} \\
\hline & & \\
\hline \multicolumn{3}{|r|}{$\left(s / q^{2}\right)^{\alpha-3}\left(-B_{w}{ }^{*} B_{w}\left(q^{2}\right)^{\alpha-2}\left(\Delta^{2} / 4 t\right)\right.$} \\
\hline $\bar{f}_{11}{ }^{-+}$ & $\left(s / q^{2}\right)^{\alpha-2}(1 / t) t B_{w}^{*} B_{w}\left(q^{2}\right)^{\alpha-1}$ & $(\alpha+1)$ \\
\hline \multicolumn{3}{|l|}{$N N \rightarrow N N:$} \\
\hline$f_{2}$ & $\left(s / p^{2}\right)^{\alpha} B_{u}^{*} B_{u}\left(p^{2}\right)^{\alpha}$ & \\
\hline$f_{4}$ & $\left(s / p^{2}\right)^{\alpha-1} t B_{v}^{*} B_{v}\left(p^{2}\right)^{\alpha-1}$ & \\
\hline$f_{6}$ & $\left(s / p^{2}\right)^{\alpha-1}(\sqrt{ } t) B_{u}{ }^{*} B_{v}\left(p^{2}\right)^{\alpha-1} p$ & \\
\hline$f_{3}$ & $\left(s / p^{2}\right)^{\alpha-2} t B_{v}^{*} B_{v}\left(p^{2}\right)^{\alpha-1}$ & \\
\hline
\end{tabular}

Because of certain phase ambiguities, we have checked factorization only of the absolute magnitudes of daughter- and conspirator trajectory residues. See also footnote a, Table IV.

structure. It has been shown by Frautschi and Jones ${ }^{16}$ that these may all be put in the following form (with $\left.\Delta_{a c} \Delta_{b \dot{d}}>0\right)$ :

$$
\begin{array}{r}
(\sqrt{ } t)^{|\lambda-\mu|}\left[\left(\bar{f}_{34,12}+\bar{f}_{-3-4,12}\right)+\left(\bar{f}_{34,12}-\bar{f}_{-3-4,12}\right)\right] \\
\sim \text { const },
\end{array}
$$

which implies (at $t=0$ )

$$
(\sqrt{ } t)^{|\lambda|+|\mu|}\left[\left(\bar{f}_{\mu, \lambda}+\vec{f}_{-\mu, \lambda}\right) \pm\left(\bar{f}_{\mu, \lambda}-\bar{f}_{-\mu, \lambda}\right)\right]=0
$$

for $|\lambda+\mu| \gtrless|\lambda-\mu|$. For $\pi \pi \rightarrow V V$, this gives the relation

$$
\left(t \bar{f}_{11}+\right)_{t=0}=-\left(t \bar{f}_{11}\right)_{t=0} .
$$

The conspiracy relation is said to be satisfied by conspiracy if any of the regularized PCHA involved in the relation are nonzero at $t=0$. Otherwise (when each term separately vanishes at $t=0$ ) the relation is said to be satisfied by evasion. ${ }^{30}$

\section{CONSEQUENCES OF SINGLE-FAMILY EXCHANGE}

In Sec. II we demonstrated that for any single leading Regge-pole exchange, a series of daughter trajectories is

\footnotetext{
${ }^{30} \mathrm{It}$ may seem at first that the factors of $i$ introduced by certain conspiracy relations indicate phases for Regge residues at variance with standard assumptions (reality for meson residues below the physical cut, etc.). If the amplitudes are examined carefully, however, it can be seen that the kinematic factors for the two $f$ 's involved differ by a term like $\left(t-4 M^{2}\right)^{1 / 2}$; perturbation-theory examples show that in all cases the additional $i$ comes from evaluation of a term like this at $t=0$. These terms are removed from the amplitude before dispersion relations can be written and hence do not influence the phase of the dynamical residue function.
}

necessary and sufficient to restore proper analyticity at $t=0$ to the individual helicity amplitudes. In that treatment, we completely neglected the influence of $t=0$ constraints between different PCHA. These constraints are considered in the present section. We work through a series of examples (the reactions $N N \rightarrow N N$, $\pi N \rightarrow V N$, and $\pi \pi \rightarrow V V$ ), separating possible cases according to the quantum numbers of the parent trajectory. This separation allows us to compare with the $O(4)$ results of Freedman and Wang ${ }^{4}$ (Sec. VI). Our chief conclusion is that the set "parent+daughters" is sufficient to satisfy $t=0$ constraint equations, provided that the parent residue is appropriately chosen. ${ }^{31}$ This provides some insight into the possible applications of FW class-I and class-II conspiracies, especially in EU and UU processes. (See Sec. VI.)

At the same time, we are interested in the question of factorization ${ }^{32}$ for the daughter residues. $A$ priori, if (at $t=0$ ) there is a single $t$-channel state with definite $P$ and $C$ (or $G$ ) at each value of $J=\alpha(0)-n$, then one expects the residue of such a state to factorize. Hence we do expect the daughters of a single- or double-parity family (corresponding to one $M=0$ or $M=1$ Lorentz pole) to factorize; these are the families we have been considering (rather than a linear mixture of several families). If the daughter residue did not factorize, one would have difficulty associating it with a single Regge

${ }^{31}$ This conclusion has been reached independently, using a different method, by J. C. Taylor, Oxford University Report, 1967 (unpublished).

${ }^{32}$ M. Gell-Mann, Phys. Rev. Letters 8, 263 (1962); V. N. Gribov and I. Ya. Pomeranchuk, ibid. 8, 343 (1962). 
trajectory; this would change the dynamical role played by such daughters.

We wish to see under what circumstances the daughter residues determined by the conspiracy and singularity-removal constraints necessarily factorize and in what cases the factorization requirement is an additional constraint one imposes on the residues..$^{33}$ In the latter case, the analyticity constraints do not fix (all terms in) the residue (e.g., because of evasion in the parent residue) and factorization helps remove some of the freedom in such residues. However, we expect factorization never to be inconsistent with the analyticity requirements.

\section{A. Leading Trajectory Has Quantum Numbers $\boldsymbol{P}=\boldsymbol{C}=(-\mathbf{1})^{J}$ (See Table II)}

\section{Contribution to the Process $\pi \pi \rightarrow V V$}

The general features of UU scattering have been discussed in Sec. II C3. $P=(-1)^{J}$ trajectories cannot couple to helicity- 0 states of the $\pi V$ system. Hence this exchange will contribute only to the $t$-channel helicity amplitudes $f_{10,10^{t}}$ and $f_{10,-10^{t}}$ with dominant contribution in the PCHA

$$
\begin{aligned}
\bar{f}_{11}+=\bar{f}_{10,10}-\bar{f}_{-10,10} & \\
& =(2 \alpha+1)\left(T_{10,10^{\alpha}}-T_{-10,10^{\alpha}}\right) e_{11}{ }^{\alpha^{+}}
\end{aligned}
$$

and minor contribution to $\bar{f}_{11^{-}}$. Assuming that

so that

$$
\left(T_{10,10^{\alpha}}-T_{10,-10^{\alpha}}\right) \sim(p q)^{\alpha-1} \sim(1 / t)^{\alpha-1}
$$

$$
\bar{f}_{11}+\sim(p q)^{\alpha-1}(z)^{\alpha-1} \sim s^{\alpha-1}
$$

(as happens in perturbation theory ${ }^{34}$ ), we see that contributions of daughter trajectories are necessary to reduce the singularity near $t=0$ of coefficients of lower powers of $s$. No power of $s$ may have a coefficient more singular than $1 / t\left[=(1 / \sqrt{ } t)^{|\lambda|+|\mu|}\right]$ to remain consistent with the singularity structure of helicity amplitudes deduced from the crossing matrix. ${ }^{35}$

When the daughter-trajectory contributions are included, we then have

$$
\begin{aligned}
\sum \bar{f}_{11}+ & =\sum_{n=0}^{\infty}(2 \alpha-2 n+1)\left(T_{10,10^{\alpha-n}}-T_{10,-10^{\alpha-n}}\right) e_{11}{ }^{(\alpha-n)^{+}} \\
& =\sum_{n=0}^{\infty} 2(2 \alpha-2 n+1) T_{10,10^{\alpha-n}} e_{11}^{(\alpha-n)^{+}}
\end{aligned}
$$

as the contribution of our $P=C=(-1)^{J}$ trajectory and its family to the dominant PCHA. This implies a

\footnotetext{
${ }^{33}$ This portion of the paper provides an answer to the questions raised in unpublished portions of California Institute of Technology Synchrotron Laboratory report CALT-68-142 by $\mathrm{S}$. Frautschi and L. Jones.

${ }_{34}$ See Appendix $\mathrm{D}$.

${ }^{35}$ Should $\left(T_{10,10^{\alpha}}-T_{10,-10^{\alpha}}\right)$ be less singular than $(1 / t)^{\alpha-1}$, fewer daughters would be required. However, in this case the $s^{\alpha-1}$ would be multiplied by unnecessary powers of $t$. We wish to impose only the minimum constraints of analyticity. Therefore we do not assume a zero in the coefficient of $s^{\alpha-1}$.
}

contribution to the minor PCHA

$$
\begin{aligned}
\sum \bar{f}_{11}- & =\sum_{n=0}^{\infty}(2 \alpha-2 n+1)\left(T_{10,10^{\alpha-n}-}-T_{\left.10,-10^{\alpha-n}\right) e_{11}{ }^{(\alpha-n)-}}\right. \\
& =\sum_{n=0}^{\infty} 2(2 \alpha-2 n+1) T_{10,10^{\alpha-n}} e_{11}{ }^{(\alpha-n)-}
\end{aligned}
$$

This amplitude is allowed a singularity $1 / t$ (see Table I), and expansion of Eq. (4.3) with the $T_{10,10^{\alpha}}$ chosen above will clearly give a singularity of this order.

In Appendix $\mathrm{C}$ we display a proof that choice of the daughters for proper analyticity of one PCHA ensures proper analyticity of the other, and after this choice of daughters, the $t=0$ constraint equation relating these two amplitudes, Eq. (3.8), is automatically satisfied. Hence no additional restrictions are imposed by the $t=0$ constraints.

Note that the odd daughters in this family have $P=+(-1)^{J}, C=-(-1)^{J}$, and hence cannot couple to the $N \bar{N}$ system, but they do couple to $\pi V$ and are needed to regularize the $\pi \pi \rightarrow V V$ amplitudes. In order to separate the parent and daughter contributions in Table II, we have written each $\pi \pi \rightarrow V V$ PCHA as a sum of two amplitudes with opposite charge conjugation behavior-viz., $\bar{f}_{11}{ }^{+}=\bar{f}_{11}{ }^{++}+\bar{f}_{11}{ }^{+-}, \bar{f}_{11}{ }^{-}=\bar{f}_{11}{ }^{++}+\bar{f}_{11}{ }^{--}$. Clearly, the minor-parity part of $\tilde{f}_{11}^{-}$receives a contribution from the first daughter pole.

It is interesting that the form of fully regularized amplitudes requires some revision of fitting formulas currently in vogue. We see that (with $k=[(\alpha-1) / \alpha]$ $\left.\times \Delta_{d b} \Delta_{c a}\right)$

$$
\begin{aligned}
& \left(\bar{f}_{10,10}-\bar{f}_{10,-10}\right) \sim s^{\alpha-1}+(k / t) s^{\alpha-2}, \\
& \left(\bar{f}_{10,10}+\bar{f}_{10,-10}\right) \sim-(k / t) s^{\alpha-2} .
\end{aligned}
$$

Hence, although $\bar{f}_{10,10} \approx-\bar{f}_{10,-10}$ to order $s^{\alpha-1}$, the next terms may become important in very low- $t$ fits, or for $\theta_{s}=0$ when $t_{\min } \approx-\left(m_{V}{ }^{2}-m_{\pi}^{2}\right)^{2} / s$. A similar situation occurs for all UU processes $(\pi N \rightarrow \rho \Delta, \gamma N \rightarrow K \Lambda$, etc.). Considering the present status of Regge fitting, it is not clear whether this modification will result in any significant change in data interpretation.

\section{Contribution to the Process $\pi N \rightarrow V N$}

The odd daughters of the family do not couple to any of the amplitudes. The leading trajectory and its even daughters contribute to $\bar{f}_{01}{ }^{++}, \bar{f}_{11}{ }^{++}$, and $\bar{f}_{11}{ }^{--}$(defined in Table I). We will discuss explicitly only regularization of $\bar{f}_{11}{ }^{++}$and $\bar{f}_{11}{ }^{--}$by daughters; the treatment of $\bar{f}_{01}{ }^{++}$(since it enters no conspiracy equation) is exactly as in Sec. II.

The amplitudes can be written as

$$
\begin{aligned}
& \sum \bar{f}_{11^{++}}=\sum_{n \text { even }}^{\infty}(2 \alpha-2 n+1) T_{10,1 / 2-1 / 2}{ }^{\alpha-n} e_{11}{ }^{(\alpha-n)+}, \\
& \sum \bar{f}_{11}{ }^{--}=\sum_{n \text { even }}^{\infty}(2 \alpha-2 n+1) T_{10,1 / 2-1 / 2}{ }^{\alpha-n} e_{11}{ }^{(\alpha-n)-} .
\end{aligned}
$$


TABLE III. Residues of parent- and first-daughter trajectories in a family with single leading $P=C=-(-1)^{J}$ pole. See Table-II caption for an explanation of the notation and interpretation of the entries.

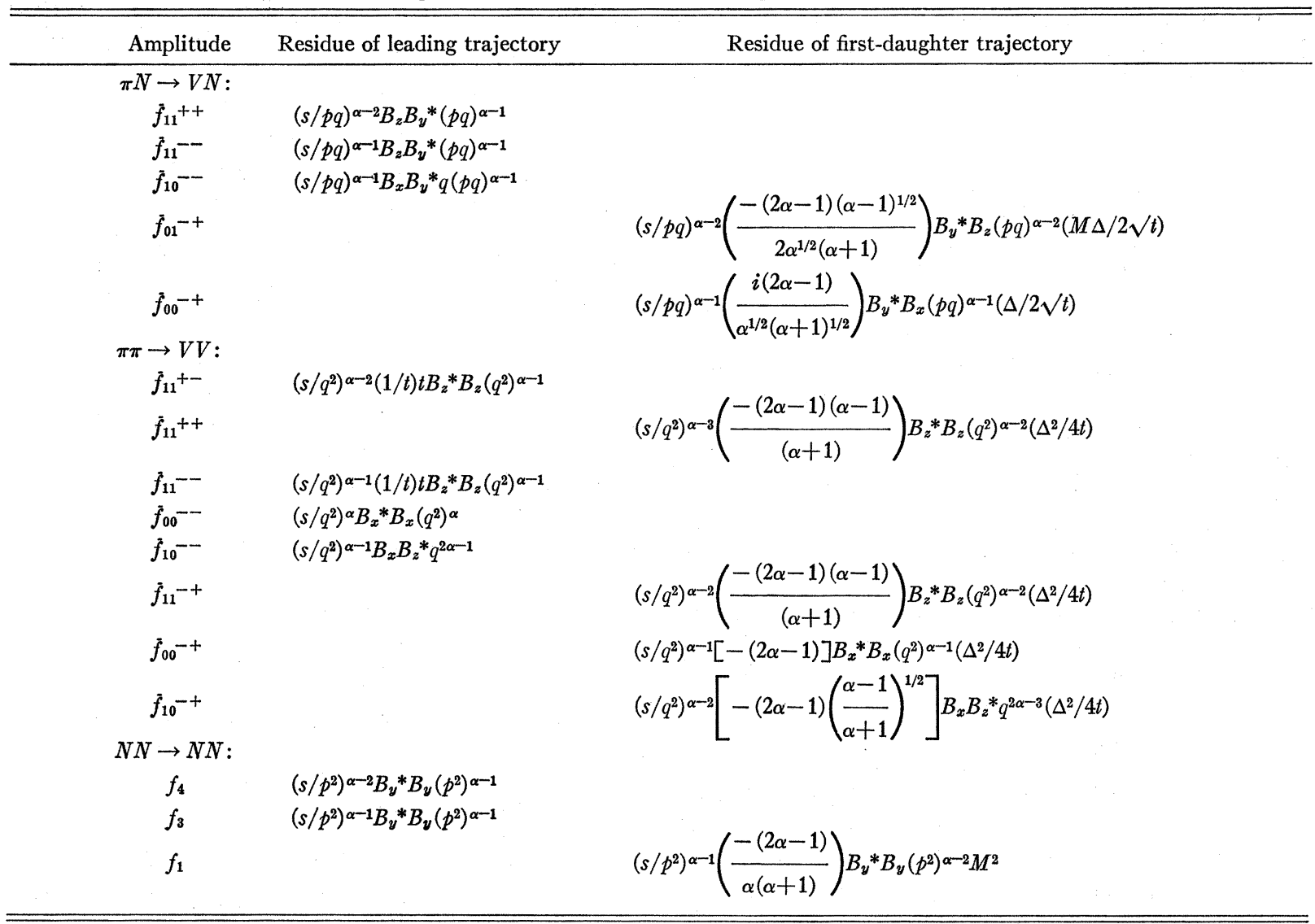

$\bar{f}_{11}--$ is allowed no kinematic singularity at $t=0$. Hence we require $T_{10,1 / 2-1 / 2}^{\alpha} \sim(1 / \sqrt{ } t)^{\alpha-2}$ near $t=0$. This behavior, plus the choice of daughters to regularize $\bar{f}_{11}--$, automatically regularizes $\tilde{f}_{11}{ }^{++}$and ensures that it satisfies the $t=0$ constraint, Eq. (3.5), by evasion. This is proved in Appendix B. Notice that an evasive behavior for $\bar{f}_{11}{ }^{++}$is necessary because $\bar{f}_{01}{ }^{-+}$receives no contributions from this family.

\section{Contribution to the Process $N N \rightarrow N N$}

As we saw in Sec. II, no daughters are required for $\mathrm{EE}$ processes. The $t=0$ constraint, Eq. (3.2b), is satisfied by evasion; this must be put in by hand if only analyticity properties are considered.

\section{Implications of Factorization of the Leading Trajectory (See Table II)}

If it is assumed that the residues of the parent trajectory satisfy the factorization theorem for the definite $P$ and $C$ parts of each amplitude, additional information may be obtained. In particular:

The evasive behavior required (in the leading power of $s$ ) by $t=0$ constraints in the amplitudes $\bar{f}_{11}++$ $(\pi \pi \rightarrow V V), \bar{f}_{11}++(\pi N \rightarrow V N)$, and $f_{4}(N N \rightarrow N N)$ is consistent with factorization; evasion in $\pi \pi \rightarrow V V$ and $\pi N \rightarrow V N$ would require evasion in $N N \rightarrow N N$. If the successive even daughters all factorize, then their appearance in the unequal-mass processes ensures their appearance in $N N \rightarrow N N$ even though they are not needed here for regularization..$^{36}$ As the odd daughters appear in only one of the three processes, nothing is learned about their factorization.

\section{B. Leading Trajectory Has Quantum Numbers $P=C=-(-1)^{J}$ (See Table III)}

\section{Contribution to the Process $\pi \pi \rightarrow V V$}

The members of this family contribute to each of the four amplitudes listed in Table I. The discussion for $\bar{f}_{11}{ }^{+}$and $\bar{f}_{11}-$ is identical to that in part A with $\bar{f}_{11}{ }^{+}$ now playing the role of dominant PCHA. The leading trajectory can now also contribute to $\bar{f}_{00}{ }^{-}$and $\bar{f}_{10}{ }^{-}$; but each of these amplitudes may be separately regularized by daughters (à $l a$ Sec. II) and both are free of linear constraints at $t=0$.

${ }^{36}$ Note that for even daughters the most singular parts of the EU and UU residues are completely determined by analyticity, when the parent residues are nonevasive. These have $t=0$ behavior which obeys factorization; the coefficients then determine the EE residue. 


\section{Contribution to $\pi N \rightarrow V N$}

The structure is rather different from that for a leading $P=C=(-1)^{J}$ exchange. Now the PCHA $\bar{f}_{11}$ contains the "dominant" $e_{11}{ }^{\alpha+}$ term. To obtain the leading term $s^{\alpha-1}$ free of kinematic singularities, we require $T_{10,1 / 2-1 / 2^{\alpha}} \sim(1 / \sqrt{ } t)^{\alpha-1}$. This is also what is found in perturbation theory. ${ }^{34}$ This behavior for $T^{\alpha}$ produces a $1 / t^{1 / 2}$ singularity in

$$
\sum \bar{f}_{11}{ }^{++}=\sum_{n}(2 \alpha-2 n+1) T_{10,1_{i} / 2-1 / 2^{\alpha-n}} e_{11}^{(\alpha-n)-}
$$

which cannot be removed by regularization with daughters. A singularity of this type is allowed by Wang's analysis in this PCHA, but the constraint equation (3.5) (which connects $\bar{f}_{11}++$ to $\bar{f}_{01}{ }^{+}$) must also be satisfied. The $\bar{f}_{01}{ }^{-}$amplitude receives contributions only from $P=-C=-(-1)^{J}$ exchanges. Thus in order to satisfy all constraint equations in this process without evasion, a contribution from $P=-C=-(-1)^{J}$ exchange is needed.

The discussion in Sec. II B shows that these quantum numbers correspond to those of the first daughter of our leading trajectory. The method of Reggeization explained in Sec. II shows that the energy dependence given to $\bar{f}_{01}{ }^{-+}$from this first daughter would be exactly right to satisfy the $t=0$ constraint equation (3.5) we have been considering. This makes it plausible that the conspirator needed is in fact the same object which plays the role of the first daughter in UU (e.g., $\pi \pi \rightarrow$ $V V$ ) reactions. The discussion in Sec. IV B 4 (below) demonstrates that this interpretation is consistent with factorization at $t=0$.

Likewise, the fully regularized contribution to $\bar{f}_{10}-$ requires a contribution to $\bar{f}_{00}{ }^{-+}$to satisfy the constraint equation (3.4). Although we could satisfy this equation by evasion and thus avoid the necessity for contributions from the first daughter, we would like to avoid extra zeros whenever possible. In addition, perturbation theory again makes the conspiring course seem a natural one. ${ }^{34}$

The daughter entries given in Table III for $\pi N \rightarrow V N$ have been determined from the conspiracy relations, Eqs. (3.4) and (3.5).

Hence the case (B) of unnatural-parity exchange with $P=C$ differs considerably from the case (A) of naturalparity exchange. For natural-parity exchange alone, we saw that only an evasive solution to the $\pi N \rightarrow V N$ conspiracy equations is possible. For $P=C=-(-1)^{J}$ exchange, a conspiratorial solution is possible provided a trajectory with the quantum numbers of the first daughter is allowed to contribute.

\section{Contribution to $N N \rightarrow N N$}

The leading trajectory contributes to the dominantparity part of $f_{3}$ and the minor-parity part of $f_{4}$. Clearly, a contribution from a trajectory with the quantum numbers of the first daughter allows a nonevasive solution of the conspiracy relation, Eq. (3.2). The entry given in Table III for $f_{1}$ follows from this.

\section{Implications of Factorization of the Leading Trajectory}

Imposition of the factorization condition on the residues of the leading trajectory leads to the dominant contributions to PCHA listed in Table III, column 1. These amplitudes in turn require dominant contributions from the first daughter (in $\pi \pi \rightarrow V V$ ) and from the conspirator (in $\pi N \rightarrow V N$ and $N N \rightarrow N N$ ) which are shown in column 2 . Notice that the residues shown in column 2 also factorize. It is therefore plausible that the conspirator and the daughter in fact arise from the same source and should be treated as one pole (at $t=0$ ). With this interpretation, we have found that the first daughter has a factorizing residue if the residue of the leading trajectory factorizes. ${ }^{37,38}$

\section{Conclusions}

The $t=0$ analyticity requirements impose a definite structure on the contributions of $P=C=-(-1)^{J}$ exchange. Either the trajectory evades, or it must be accompanied by a $P=-C=-(-1)^{J}$ exchange one unit lower in spin. This exchange has the right quantum numbers to be the first daughter of the leading trajectory. This interpretation is supported by application of the factorization of Regge residues. As the lower exchange must also be regularized by daughters, all odd daughters are brought into play.

In Sec. VI we shall consider the two other possible single-parity families [with leading pole $P=-C$ $=-(-1)^{J}$ or $\left.P=-C=+(-1)^{J}\right]$ and show that they evade when coupled to the $N \bar{N}$ system.

\section{PARITY-DOUBLED LEADING TRAJECTORIES (WITH $C=+(-1)^{J}$ )}

In Sec. II we pointed out some general features of the analytic structure of EU and UU amplitudes when they receive contributions from a parity-doubled family of poles. Now we look at the specific processes $\pi \pi \rightarrow V V$, $\pi N \rightarrow V N, N N \rightarrow N N$ and consider the exchange of two opposite-parity leading trajectories with $C=+(-1)^{J}$. As before, our concern is to satisfy all $t=0$ analyticity constraints. [The parity-doubled family with leading $C=-(-1)^{J}$ trajectories will be discussed in Sec. VI.]

${ }^{37}$ At the present state of understanding, the question of whether or not the singularity-removing pieces necessarily correspond to dynamical poles has not been conclusively answered. It is not entirely clear, therefore, that the factorization found above (for daughters) should necessarily hold anywhere other than $t=0$.

${ }^{38}$ Notice that the "kinematic factor" $1 / \sqrt{ } t$ in $\tilde{f}_{00}{ }^{+}(\pi N \rightarrow V N)$ is balanced (in the factorization condition equation) by the $1 / t$ singularity of a daughter residue in $f_{00 ; 00}(\pi \pi \rightarrow V V)$. This is a case in which residues factor at $t=0$ even though the Wang $K(t)$ 's do not; it suggests a solution to at least some of the factorization problems studied by Leader (Ref. 10). 
TABLE IV. Residues of parent- and first-daughter trajectories in a family with parity-doubled leading poles, $P=C=(-1)^{J}$ and

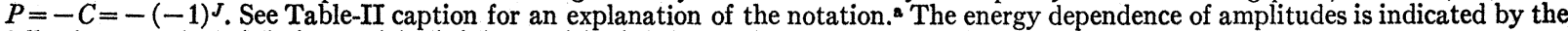
following notation: $(s / p q)^{\alpha-n}=(\tilde{n}) ;\left(s / q^{2}\right)^{\alpha-n}=(n) ;\left(s / p^{2}\right)^{\alpha-n}=(n)$.

\begin{tabular}{|c|c|c|c|}
\hline Amplitude & $\begin{array}{c}\text { Residue of leading } \\
P=-(-1)^{J} \text { trajectory }\end{array}$ & $\begin{array}{l}\text { Residue of leading } P=(-1)^{J} \\
\text { trajectory }\end{array}$ & $\begin{array}{l}\text { Residue of first } P=-(-1)^{J} \\
\text { daughter trajectory }\end{array}$ \\
\hline \multicolumn{4}{|l|}{$\pi N \rightarrow V N:$} \\
\hline$\hat{f}_{01}++$ & & (i) $\quad B_{r} B_{e}{ }^{*}(p q)^{\alpha-1} p$ & \\
\hline$\tilde{f}_{11}++$ & & (i) $-i\left(\frac{\alpha+1}{\alpha}\right)^{1 / 2}\left(\frac{1}{\sqrt{ } t}\right) B_{r} B_{t}^{*}(p q)^{\alpha-1} p$ & 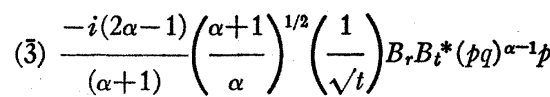 \\
\hline$\tilde{f}_{11}^{--}$ & & $(\overline{2})-i\left(\frac{\alpha+1}{\alpha}\right)^{1 / 2}\left(\frac{1}{\sqrt{ } t}\right) B_{r} B_{t}^{*}(p q)^{\alpha-1} p$ & (2) $\frac{-i(2 \alpha-1)}{(\alpha+1)}\left(\frac{\alpha+1}{\alpha}\right)^{1 / 2}\left(\frac{1}{\sqrt{ } t}\right) B_{r} B_{t}^{*}(p q)^{\alpha-1} p$ \\
\hline $\bar{f}_{10^{--}}$ & & & (2) $\quad \mathbb{R}_{1} \mathrm{~b}$ \\
\hline$\tilde{f}_{01}{ }^{-+}$ & (i) $(1 / \sqrt{ } t) B_{r} B_{t}^{*}(p q)^{\alpha-1} p$ & & \\
\hline$\tilde{f}_{00^{-+}}$ & $(\overline{0})(\sqrt{ } t) B_{s} B_{t}^{*}(p q)^{\alpha}$ & & \\
\hline \multicolumn{4}{|l|}{$\pi \pi \rightarrow V V:$} \\
\hline$f_{11}+-$ & & & (3) $-(2 \alpha-1)(1 / t) B_{r}{ }^{*} B_{r}\left(q^{2}\right)^{\alpha-2}\left(\Delta^{2} / 4 t\right)$ \\
\hline$\tilde{f}_{11}++$ & (2) $(1 / t) B_{r}^{*} B_{r}\left(q^{2}\right)^{\alpha-1}$ & (1) $-(1 / t) B_{r}^{*} B_{r}\left(q^{2}\right)^{\alpha-1}$ & \\
\hline$\tilde{f}_{11}^{--}$ & & & (2) $-(2 \alpha-1)(1 / t) B_{r}^{*} B_{r}\left(q^{2}\right)^{\alpha-2}\left(\Delta^{2} / 4 t\right)$ \\
\hline$\tilde{f}_{00}-$ & & & (1) $\quad \mathbb{R}_{2} \mathrm{~b}$ \\
\hline$\vec{f}_{10^{--}}$ & & & (2) $-(2 \alpha-1)\left(\frac{\alpha-1}{\alpha+1}\right)^{1 / 2} B_{r}^{*} B_{s} q^{2 \alpha-3}\left(\Delta^{2} / 4 t\right)$ \\
\hline$\dot{f}_{11}{ }^{-+}$ & (1) $(1 / t) B_{r}^{*} B_{r}\left(q^{2}\right)^{\alpha-1}$ & (2) $-(1 / t) B_{r}^{*} B_{r}\left(q^{2}\right)^{\alpha-1}$ & \\
\hline$\tilde{f}_{00}{ }^{-+}$ & (0) $t B_{s}^{*} B_{s}\left(q^{2}\right)^{\alpha}$ & & \\
\hline$\dot{f}_{10}{ }^{+}$ & (1) $B_{r}^{*} B_{s} q^{2 \alpha-1}$ & & \\
\hline \multicolumn{4}{|l|}{$N N \rightarrow N N:$} \\
\hline$f_{2}$ & & () $t B_{e}^{*} B_{e}\left(p^{2}\right)^{\alpha}$ & \\
\hline$f_{4}$ & & (1) $[(\alpha+1) / \alpha] B_{t}^{*} B_{t}\left(p^{2}\right)^{\alpha}$. & $\mathbb{R}_{3} \mathrm{~b}$ \\
\hline$f_{5}$ & & (1) $[(\alpha+1) / \alpha]^{1 / 2}(\sqrt{ } t) B_{t}^{*} B_{e}\left(p^{2}\right)^{\alpha}$ & \\
\hline$f_{3}$ & & (2) $[(\alpha+1) / \alpha] B_{t}^{*} B_{t}\left(p^{2}\right)^{\alpha}$ & $a_{3} b$ \\
\hline$f_{1}$ & (0) $B_{t}^{*} B_{t}\left(p^{2}\right)^{\alpha}$ & & \\
\hline
\end{tabular}

If a residue can be obtained only by factorization, its phase is in many cases undetermined. We have not indicated such possible phase ambiguities in the Tables.

$\mathrm{b}$ This residue, though not fixed by analyticity requirements, is determined if we assume that the residues of the first daughter trajectory factorize. See Sec. $\mathbf{V}$ for the value thus determined.

In the examples discussed in Sec. IV, certain of the constraint equations at $t=0$ could be satisfied by conspiracy only in lower powers of $s$. For example, the dominant power of $s$ in the PCHA for UU reactions always has a singularity of the form

$$
(1 / \sqrt{ } t)^{\lambda^{\prime}}, \quad \text { where } \lambda^{\prime}=\min (|\lambda-\mu|,|\lambda+\mu|),
$$

provided exchanges of only one parity type are allowed. This leads us to conjecture the existence of a new method of satisfying the conspiracy equations: Allow two trajectories with the same $\alpha$ at $t=0$ but of opposite parity types (so that each will contribute the dominant term to a different PCHA). In this case, the dominant power of $s$ in each PCHA may have its maximum singularity, $(1 / \sqrt{ } t)^{\lambda^{\prime \prime}}$ where $\lambda^{\prime \prime}=\max (|\lambda-\mu|$, $|\lambda+\mu|)$, provided the residues of the conspiring trajectories are such that Eq. (3.6) is satisfied. In our analyticity approach, there appears to be no other way to "derive" the existence of this type of pole structure. It is just a natural solution to the constraint equations.
As we have shown for single-parity families, once we assume that the top-lying trajectories exist, the lowerlying daughters are needed to assure the proper analyticity of the individual PCHA.

As we noted in Sec. II, close examination of UU processes makes it clear that the pattern of daughters required for singularity cancellation in this case is rather different from a superposition of two single-parity types. For example, in $\pi \pi \rightarrow V V$,

$$
\begin{array}{r}
\bar{f}_{11}{ }^{-}=(2 \alpha+1) T^{+}{ }_{10,10}{ }^{\alpha} e_{11}{ }^{\alpha^{+}}+(2 \alpha+1) T^{-}{ }_{10,10^{\alpha}} e_{11}{ }^{\alpha^{-}} \\
+ \text {daughters, } \\
\bar{f}_{11}{ }^{+}=(2 \alpha+1) T^{+}{ }_{10,10}{ }^{\alpha} e_{11}{ }^{\alpha^{-}}+(2 \alpha+1) T^{-}{ }_{10,10} e_{11}{ }^{\alpha^{+}} \\
+ \text {daughters. }
\end{array}
$$

Our new conspiracy is marked by $T^{\alpha+} \sim C / t^{\alpha} \sim-T^{\alpha-}$ for the most singular piece. The contribution of the first daughter $T_{10,10}^{(\alpha-1)+} e_{11}^{(\alpha-1)+}$ of the unnaturalparity parent pole to $\bar{f}_{11}-$ must then correct a singularity of the form $t^{-2} s^{\alpha-2}$ arising from parents of both parities. 
This is quite different from the cases discussed in Sec. IV, in which daughters were required to remove singularities of a unique parity. However, for UU amplitudes with $\lambda$ or $\mu=0$, the pattern is similar to the cases in Sec. IV, since then only a single-parity type contributes.

This kind of collaboration is also easily seen in EU processes such as $\pi N \rightarrow V N$. (See Sec. II C 2.) The appropriate conspiracy equation here is Eq. (3.5). Thus the contribution of the leading natural-parity trajectory to $\bar{f}_{11}{ }^{++}$has a $1 / \sqrt{ } t$ kinematic singularity

$$
\sim \frac{(p q)^{\alpha-1}}{\sqrt{ } t}\left(\frac{2 \alpha}{\alpha+1} \frac{\Gamma\left(\alpha+\frac{1}{2}\right)}{\Gamma(\alpha+1)}\right)\left(\frac{s}{p q}\right)^{\alpha-1}
$$

It contributes to the corresponding minor-parity part of the amplitude $\bar{f}_{11}-$ - something of the form

$$
\sim \frac{(p q)^{\alpha-1}}{\sqrt{ } t}\left(\frac{4(1-\alpha)}{\alpha+1} \frac{\Gamma\left(\alpha+\frac{1}{2}\right)}{\Gamma(\alpha+1)}\right)\left(\frac{s}{p q}\right)^{\alpha-2},
$$

which has a $1 / t$ singularity at $t=0$. However, the amplitude $\bar{f}_{11^{-}}-$is not allowed to have such a kinematic singularity; hence it must be cancelled by another contribution. The first "daughter" of the leading unnatural-parity exchange has the right quantum numbers to contribute here; we therefore require its contribution to cancel the singularity induced by the natural-parity parent.

This behavior seems to indicate that in a conspiracy of this sort, where parity is not a good quantum number for the combined parents, it should not be considered important in classifying the lower poles which remove singularities.

By assigning daughter contributions in this fashion, we have been able to study the implications of factorization also in this case. The results cannot be stated as strongly as those for the single-parity exchange cases. This is due to kinematic details which prevent some of the residues of the first daughter from being entirely determined from those of the parent by the requirements of singularity cancellation. For example, consider the residue functions displayed in Table IV. The three entries $R_{i}(i=1,2,3)$ indicate residues of the first daughter which cannot be determined by singularity cancellation alone.

(a) In $\pi N \rightarrow V N$, the residue in $\bar{f}_{10}-$ is determined from that of $\bar{f}_{00}{ }^{-}$by the conspiracy condition. The leading term in $\hat{f}_{00}{ }^{-+}$, however, has a residue function with evasive behavior. This means that the residue of the second daughter (which is the first daughter to appear in the same amplitude as the parent) is not determined by singularity cancellation, because the singularity of the parent to this order of $s$ is allowed. Thus the sum of parent and second daughter is not determined, and one cannot determine the residue of the first daughter by the conspiracy condition. (b) In $\pi \pi \rightarrow V V, \bar{f}_{00^{-+}}$contains an extra factor of $t$ in the residue of the parent pole (to ensure factorization among the parent residues in this reaction). This means that the residue of the first daughter is not determined by singularity cancellation. ${ }^{39}$

(c) In $N N \rightarrow N N$, the residue of the first daughter in $f_{3}$ cannot be determined from the conspiracy condition unless the contributions of the second daughter to $f_{1}$ and $f_{4}$ are known.

These three residues $\mathfrak{O}_{i}$ can, however, be determined from the other ones (which are obtained completely from singularity cancellation) if we assume that the residues of the first daughter satisfy factorization. The residues obtained in this fashion have proper singularity structure to play the roles necessary of them. (We examine here only the most singular portions of the residue.)

From internal factorization in $\pi \pi \rightarrow V V$, we find

$$
\mathfrak{R}_{2}=-B_{s}^{*} B_{s} q^{2 \alpha}\left(\frac{\alpha-1}{\alpha+1}\right)(2 \alpha-1) t
$$

Cross factorization of the three reactions gives

$$
R_{3}=-\frac{(2 \alpha-1)}{\alpha(\alpha+1)} p^{2 \alpha} B_{t}^{*} B_{t} .
$$

Then, using $\mathscr{R}_{2}$ and $\mathscr{R}_{3}$, one can obtain $\mathbb{R}_{1}$ by again applying cross factorization

$$
\mathfrak{R}_{1}=\frac{(\text { phase })(2 \alpha-1)(p q)^{\alpha} B_{s} B_{t}^{*} \sqrt{ } t}{(\alpha+1)}\left(\frac{\alpha-1}{\alpha}\right)^{1 / 2} .
$$

We thus conclude that factorization of the first $P=-(-1)^{J}$ daughter is consistent with the singularity structure of the amplitude. The first $P=(-1)^{J}$ daughter appears only in the one reaction $\pi \pi \rightarrow V V$, hence there is no question of factorization. The fact that we could determine all the first daughter residues from singularity removal and factorization leads one to conjecture that these principles are enough to always obtain the most singular pieces of the daughters from the parent residues. Examples in Sec. VI demonstrate that these principles are not enough to determine all daughters uniquely from the parents; for this, one requires a specific model such as Feynman diagrams or the BetheSalpeter equation.

Note that the entries in Table IV are based on the most singular terms in the residues of the leading poles. As can be seen from Eq. (2.39), the daughters calculated to cancel these terms are such that $P=(-1)^{J}$ daughters have the same residue function (up to a sign) as $P$

${ }^{39}$ This is actually a good thing: The residue of this first daughter in $\tilde{f}_{10}$ is the same as in the single-parity-exchange case (same singularities to cancel), but the residue in $\bar{f}_{11}$ is different (from discussion above). If the singularity in $\tilde{f}_{00}$ were determined by multiplying the residue for single-parity exchange by $t$, there would be no hope of factorization among $\pi \pi \rightarrow V V$ residues of the first daughter. 
TABLE V. Residues of parent- and first-daughter trajectories in a family with single leading $P=-C=(-1)^{J}$ pole. The residue of the leading pole has been divided by $(2 \alpha+1)$ and that of the first daughter by $(2 \alpha-1)$. Another satisfactory solution is to have all of the $\pi \pi \rightarrow V V$ daughter residues multiplied by $t$, with the $N N \rightarrow N N$ residues divided by $t$. See Table-II caption for an explanation of the notation. ${ }^{\mathrm{a}}$

\begin{tabular}{|c|c|c|}
\hline Amplitude & Residue of leading trajectory & Residue of first-daughter trajectory \\
\hline \multicolumn{3}{|l|}{$\pi N \rightarrow V N:$} \\
\hline$\tilde{f}_{01}{ }^{++}$ & & $(s / p q)^{\alpha-2}(2 \alpha+1)^{1 / 2}[(\alpha-1) /(\alpha+1)]^{1 / 2}(\sqrt{ } t) B_{u}{ }^{*} B_{z}(p q)^{\alpha-1}$ \\
\hline $\bar{f}_{11}++$ & & $(s / p q)^{\alpha-2}(2 \alpha+1)^{1 / 2}[(\alpha-1) /(\alpha+1)]^{1 / 2} t B_{v}^{*} B_{z}(p q)^{\alpha-2} q$ \\
\hline$\tilde{f}_{11}--$ & & $(s / p q)^{\alpha-3}(2 \alpha+1)^{1 / 2}[(\alpha-1) /(\alpha+1)]^{1 / 2} t B_{v}^{*} B_{z}(p q)^{\alpha-2} q$ \\
\hline \multicolumn{3}{|l|}{$\pi \pi \rightarrow V V:$} \\
\hline$\tilde{f}_{11}+-$ & $\left(s / q^{2}\right)^{\alpha-1}(1 / t) t B_{z}^{*} B_{z}\left(q^{2}\right)^{\alpha-1}$ & \\
\hline $\bar{f}_{11}^{++}$ & & $\left(s / q^{2}\right)^{\alpha-2}[-(2 \alpha+1)][(\alpha-1) /(\alpha+1)] B_{z}^{*} B_{z}\left(q^{2}\right)^{\alpha-1}$ \\
\hline$\tilde{f}_{11}^{--}$ & $\left(s / q^{2}\right)^{\alpha-2}(1 / t) t B_{z}^{*} B_{z}\left(q^{2}\right)^{\alpha-1}$ & \\
\hline$\tilde{f}_{11}{ }^{-+}$ & & $\left(s / q^{2}\right)^{\alpha-3}[-(2 \alpha+1)][(\alpha-1) /(\alpha+1)] B_{z}^{*} B_{z}\left(q^{2}\right)^{\alpha-1}$ \\
\hline \multicolumn{3}{|l|}{$N N \rightarrow N N:$} \\
\hline$f_{2}$ & & $\left(s / p^{2}\right)^{\alpha-1}\left[-t B_{u} * B_{u}\left(p^{2}\right)^{\alpha-1}\right]$ \\
\hline$f_{4}$ & & $\left(s / p^{2}\right)^{\alpha-2}\left[-t^{2} B_{v}^{*} B_{v}\left(p^{2}\right)^{\alpha-2}\right]$ \\
\hline$f_{5}$ & & $\left(s / p^{2}\right)^{\alpha-2}\left[-t^{1 / 2} t B_{u}{ }^{*} B_{v} p^{2 \alpha-3}\right]$ \\
\hline$f_{3}$ & & $\left(s / p^{2}\right)^{\alpha-3}\left[-t^{2} B_{v}^{*} B_{v}\left(p^{2}\right)^{\alpha-2}\right]$ \\
\hline
\end{tabular}

${ }^{2}$ See Table IV, footnote a.

$=(-)(-1)^{J}$ daughters; differences in the forms of daughters of different parity types are obtained entirely from the less singular portion of the parent residue functions. Only the most singular daughter contributions are shown in Table IV; we will not consider the details of lower singularities here.

\section{COMPARISON WITH THE $O(4)$ ANALYSIS OF FREEDMAN AND WANG}

The three types of pole structure discussed above in Secs. IV A, IV B, and V clearly correspond to the three types of conspiracy in $N N$ scattering discussed by Freedman and Wang ${ }^{4}$ : the discussion in Sec. IV A to their type I, the discussion in Sec. IV B to their type II, and the case of Sec. V to their type III. Our analysis suggests that the only difference between FreedmanWang types I and II is that the first daughters of type-I trajectories cannot couple to the $N \bar{N}$ state, whereas those of type-II trajectories can, and thus type II allows a nonevasive solution to the conspiracy relations.

The restriction that one is coupling to $N \bar{N}$ states placed other limits on the types of conspiracy Freedman and Wang could hope to find. Simple counting of quantum numbers would lead one to believe that there should be two other types of $M=0$ conspiracies [one with leading trajectory $P=-C=(-1)^{J}$ and one with leading trajectory $\left.P=-C=-(-1)^{J}\right]$, and at least one other type of $M=1$ conspiracy [with leading trajectories having $\left.C=-(-1)^{J}\right]$. It is not very difficult to demonstrate that there are reactions in which conspiracies of this type will play an important role. In the following paragraphs we discuss each of these types in turn and demonstrate that they necessarily evade in $N N$ scattering. (Only the most singular portions of the residues are considered.)

\section{A. Single-Parity Exchange with Leading Trajectory Having $P=-C=(-1)^{J}$}

The leading trajectory cannot couple to $N \bar{N}$ states; hence the contributions to $N N \rightarrow N N$ must all be given by odd daughters and these must all evade. One would expect that a pattern of contributions to $\pi \pi \rightarrow$ $V V$ similar to that of Freedman-Wang class I should be possible. However, under factorization across the three reactions (see Table V), one then obtains contributions to $\pi N \rightarrow V N$ which have the wrong singularity structure at $t=0$. It appears that leading terms in one of the reactions $\pi \pi \rightarrow V V$ and $N N \rightarrow N N$ must "doubly evade" (i.e., contain a double-zero $t^{2}$ in the residue function), and that the residue of the other reaction must "singly evade" to produce proper behavior in $\pi N \rightarrow V N$.

One could construct an EE reaction containing a conspiracy led by a pole with $P=-C=(-1)^{J}$ by the following formal device: The reaction $N N^{\prime} \rightarrow N^{\prime} N$, where $N^{\prime}$ is a particle with the mass and spin of the nucleon but with opposite parity, has the same conspiracy relation as $N N$ scattering. The parity identification of all the PCHA is, however, reversed and charge conjugation has ceased to be a good quantum number with which to identify the states (because the $N^{\prime} \bar{N}$ state is not a particle-antiparticle state). Both $C$ types of $P=(-1)^{J}$ conspiracy are then possible, with nonvanishing residues. 
TABLE VI. Residues of parent- and first-daughter trajectories in a family with single leading $P=-C=-(-1)^{J}$ pole. The residue of the leading pole has been divided by $(2 \alpha+1)$ and that of the daughter by $(2 \alpha-1)$. See Table-II caption for an explanation of the notation. ${ }^{\mathrm{s}}$

\begin{tabular}{|c|c|c|}
\hline Amplitude & Residue of leading trajectory & Residue of first-daughter trajectory \\
\hline \multicolumn{3}{|l|}{$\pi N \rightarrow V N:$} \\
\hline$\dot{f}_{11}^{++}$ & & $(s / p q)^{\alpha-3}(2 \alpha+1)^{1 / 2}[(\alpha-1) /(\alpha+1)]^{1 / 2}(\sqrt{ } t) B_{z}^{*} B_{b}(p q)^{\alpha-2} q$ \\
\hline $\bar{f}_{11}--$ & & $(s / p q)^{\alpha-2}(2 \alpha+1)^{1 / 2}[(\alpha-1) /(\alpha+1)]^{1 / 2}(\sqrt{ } t) B_{z}^{*} B_{b}(p q)^{\alpha-2} q$ \\
\hline$\dot{f}_{10}--$ & & $(s / p q)^{\alpha-2}(2 \alpha+1)^{1 / 2}(\sqrt{ } t) B_{z}^{*} B_{a}(p q)^{\alpha-2} q^{2}$ \\
\hline $\bar{f}_{01}{ }^{++}$ & $(s / p q)^{\alpha-1}(\sqrt{ } t) B_{b}^{*} B_{y}(p q)^{\alpha-1} p$ & \\
\hline$\tilde{f}_{00^{-+}}$ & $(s / p q)^{\alpha}(\sqrt{ } t) B_{a}^{*} B_{y}(p q)^{\alpha}$ & \\
\hline \multicolumn{3}{|l|}{$\pi \pi \rightarrow V V:$} \\
\hline$\tilde{f}_{11}^{+-}$ & & $\left(s / q^{2}\right)^{\alpha-3}[-(2 \alpha+1)][(\alpha-1) /(\alpha+1)] B_{b} * B_{b}\left(q^{2}\right)^{\alpha-1}$ \\
\hline$\tilde{f}_{11}++$ & $\left(s / q^{2}\right)^{\alpha-2}(1 / t) t B_{b}^{*} B_{b}\left(q^{2}\right)^{\alpha-1}$ & \\
\hline$\tilde{f}_{11}{ }^{--}$ & & $\left(s / q^{2}\right)^{\alpha-2}[-(2 \alpha+1)][(\alpha-1) /(\alpha+1)] B_{b}^{*} B_{b}\left(q^{2}\right)^{\alpha-1}$ \\
\hline$\dot{f}_{00} 0^{-}$ & & $\left(s / q^{2}\right)^{\alpha-1}[-(2 \alpha+1)] B_{a}^{*} B_{a} q^{2 \alpha}$ \\
\hline $\bar{f}_{10}{ }^{--}$ & & $\left(s / q^{2}\right)^{\alpha-2}[-(2 \alpha+1)][(\alpha-1) /(\alpha+1)]^{1 / 2} B_{a}^{*} B_{b} q^{2 \alpha-1}$ \\
\hline$\tilde{f}_{11}{ }^{-+}$ & $\left(s / q^{2}\right)^{\alpha-1}(1 / t) t B_{b} * B_{b}\left(q^{2}\right)^{\alpha-1}$ & \\
\hline$\tilde{f}_{00^{-+}}$ & $\left(s / q^{2}\right)^{\alpha} B_{a}{ }^{*} B_{a}\left(q^{2}\right)^{\alpha}$ & \\
\hline$\dot{f}_{10^{-+}}$ & $\left(s / q^{2}\right)^{\alpha-1} B_{a}^{*} B_{b} q^{2 \alpha-1}$ & \\
\hline \multicolumn{3}{|l|}{$N N \rightarrow N N:$} \\
\hline$f_{4}$ & & $\left(s / p^{2}\right)^{\alpha-3}\left[-t B_{z}^{*} B_{z}\left(p^{2}\right)^{\alpha-2}\right]$ \\
\hline$f_{3}$ & & $\left(s / p^{2}\right)^{\alpha-2}\left[-t B_{z}^{*} B_{z}\left(p^{2}\right)^{\alpha-2}\right]$ \\
\hline$f_{1}$ & $\left(s / p^{2}\right)^{\alpha} t B_{y}^{*} B_{y}\left(p^{2}\right)^{\alpha}$ & \\
\hline
\end{tabular}

a If a residue can be obtained only by factorization, its phase is in many cases undetermined. We have not indicated such possible phase ambiguities in the Tables.

\section{B. Single-Parity Exchange with Leading Trajectory Having $P=-C=-(-1)^{J}$}

At the $N \bar{N}$ vertex, trajectories such as the pion with $P=-C=-(-1)^{J}$ can couple only to states of spin 0 . This means they can couple only to amplitudes with zero helicity change at this vertex. Because of the energy dependence $\bar{f}_{\mu \lambda} \sim s^{\alpha-\Lambda}[$ with $\Lambda=\max (|\lambda|,|\mu|)]$, no trajectory of this kind can be the leading trajectory in the $N N \rightarrow N N$ conspiracy. Hence it is only in less common reactions that we can hope for $t=0$ constraint equations which allow the pion-like trajectories to be leaders in a conspiracy relation. ${ }^{40}$

For example, the conspiracy relation for $\pi \pi \rightarrow V V$, assuming $m_{\pi}=m_{V}$, is $0=\left(\bar{f}_{10,10}+\bar{f}_{10,-10}\right)+z\left(\bar{f}_{10,10}-\bar{f}_{10,-10}\right)$ $-\bar{f}_{00,00}$. This allows a solution by a trajectory family led by a pole with $P=-C=-(-1)^{J}$, e.g., $B$ or $\pi$ meson. (To avoid a restriction on $C$, we take the case where the $\pi$ and/or the $V$ is charged.)

Study of this kind of conspiracy in our three reactions shows (Table VI):

(1) All trajectories in the family evade in $N N \rightarrow N N$.

(2) The structure in $\pi \pi \rightarrow V V$ is similar to that for Freedman-Wang types I and II.

(3) Although leading powers of $\nu$ are not involved in conspiracy in $\pi N \rightarrow V N$ there is a complicated relation

40 Recent evidence indicates that the physical pion may belong to this $M=0$ Lorentz family, as was proposed earlier by R. F. Sawyer, Phys. Rev, Letters 18, 1212 (1967), between parent and first and second daughters imposed by the conspiracy.

\section{Parity-Doubled Leading Trajectories with $C=-(-1)^{J}$}

These would produce a singularity structure in the $\pi \pi \rightarrow V V$ amplitudes exactly like that displayed above for the class-III $C=+(-1)^{J}$ case. However, because the $P=-C=(-1)^{J}$ exchanges cannot couple to $N \bar{N}$ states, the contributions to the other two reactions will again be quite different from those of a leading pair with $C=(-1)^{J}$. Only the odd daughters in the $P$ $-(-1)^{J}$ sequence will play a role.

Fewer of the daughter residues can be discovered solely from singularity cancellation and conspiracy conditions in this case than in the corresponding case for parity-doubled leading trajectories of the other $C$. If, however, it is assumed in addition that the first daughters must factorize, we learn that (Table VII):

(1) All parent- and first-daughter residues must evade in $N N \rightarrow N N$.

(2) In $\pi N \rightarrow V N$, the relation between $\vec{f}_{11}{ }^{++}$and $\bar{f}_{10^{-}}{ }^{+}$is satisfied by conspiracy, but it is not possible to determine completely the individual contributions of the two first daughters in terms of the parent residue. The relation between $\bar{f}_{10}--$ and $\bar{f}_{00}{ }^{-+}$is similar to the case of $C=+(-1)^{J}$ parents in that evasive behavior of the parent residue (necessitated by factorization) 
TABLE VII. Residues of parent- and first-daughter trajectories in a family with parity-doubled leading poles, $P=C=-(-1)^{J}$ and $P=-C=(-1)^{J}$. The residues of the leading poles have been divided by $(2 \alpha+1)$ and those of the daughters by $(2 \alpha-1)$. See captions of Tables II and IV for an explanation of the notation. ${ }^{\mathbf{a}, \mathrm{b}}$

\begin{tabular}{|c|c|c|c|}
\hline Amplitude & $\begin{array}{c}\text { Residue of leading } \\
P=-(-1)^{J} \text { trajectory }\end{array}$ & $\begin{array}{l}\text { Residue of first } P=-(-1)^{J} \\
\text { daughter trajectory }\end{array}$ & $\begin{array}{l}\text { Residue of first } P=(-1)^{J} \\
\text { daughter trajectory }\end{array}$ \\
\hline \multicolumn{4}{|l|}{$\pi N \rightarrow V N:$} \\
\hline $\bar{f}_{01}{ }^{++}$ & & & (र) $(2 \alpha+1)^{1 / 2}(\sqrt{ } t) B_{f} B_{r}{ }^{*}(p q)^{\alpha-1}$ \\
\hline$\tilde{f}_{11}++$ & (2) $B_{r}^{*} B_{y}(p q)^{\alpha-1}$ & & (र) $(2 \alpha+1)^{1 / 2} B_{e} B_{r}^{*}(p q)^{\alpha-2} q$ \\
\hline$\tilde{f}_{11}^{--}$ & (i) $B_{r}^{*} B_{y}(p q)^{\alpha-1}$ & & $(\overline{3})(2 \alpha+1)^{1 / 2} B_{e} B_{r}^{*}(p q)^{\alpha-2} q$ \\
\hline $\begin{array}{l}\tilde{f}_{10^{--}} \\
\dot{f}_{01^{-+}}\end{array}$ & (i) $t B_{s}^{*} B_{y}(p q)^{\alpha-1} q$ & $(\overline{2})(2 \alpha+1)^{1 / 2} B_{r}{ }^{*} B_{x}(p q)^{\alpha-1}$ & \\
\hline$\tilde{f}_{00^{-+}}$ & & (i) $(2 \alpha+1)^{1 / 2}[(\alpha-1) /(\alpha+1)]^{1 / 2} t B_{s}^{*} B_{x}(p q)^{\alpha-1} q$ & \\
\hline \multicolumn{4}{|l|}{$\pi \pi \rightarrow V V:$} \\
\hline $\bar{f}_{11}^{+-}$ & (2) $(1 / t) B_{r}^{*} B_{r}\left(q^{2}\right)^{\alpha-1}$ & & \\
\hline$\tilde{f}_{11}^{++}$ & & (3) $-(2 \alpha+1)(1 / t) B_{r}^{*} B_{r}\left(q^{2}\right)^{\alpha-1}$ & (2) $-(2 \alpha+1)(1 / t) B_{r}^{*} B_{r}\left(q^{2}\right)^{\alpha-1}$ \\
\hline$\tilde{f}_{11}^{--}$ & (1) $(1 / t) B_{r}^{*} B_{r}\left(q^{2}\right)^{\alpha-1}$ & & \\
\hline$\tilde{f}_{00^{--}}$ & (0) $t B_{s}^{*} B_{s}\left(q^{2}\right)^{\alpha}$ & & \\
\hline$\tilde{f}_{10^{--}}$ & (1) $B_{r}{ }^{*} B_{s}\left(q^{2}\right)^{\alpha-1} q$ & & \\
\hline$\tilde{f}_{11}^{-+}$ & & (2) $-(2 \alpha+1)(1 / t) B_{r}^{*} B_{r}\left(q^{2}\right)^{\alpha-1}$ & (3) $-(2 \alpha+1)(1 / t) B_{r}{ }^{*} B_{r}\left(q^{2}\right)^{\alpha-1}$ \\
\hline$\tilde{f}_{00^{-+}}$ & & (1) $-(2 \alpha+1)[(\alpha-1) /(\alpha+1)] t B_{s}^{*} B_{s}\left(q^{2}\right)^{\alpha}$ & \\
\hline$\dot{f}_{10}{ }^{-+}$ & & (2) $-(2 \alpha+1)[(\alpha-1) /(\alpha+1)]^{1 / 2} B_{r}{ }^{*} B_{s} q^{2 \alpha-1}$ & \\
\hline \multicolumn{4}{|l|}{$N N \rightarrow N N:$} \\
\hline$f_{2}$ & & & $(\underline{1})-t^{2} B_{f}^{*} B_{f}\left(p^{2}\right)^{\alpha-1}$ \\
\hline$f_{4}$ & (2) $t B_{y}^{*} B_{y}\left(p^{2}\right)^{\alpha-1}$ & & $(\underline{2})-t B_{e}^{*} B_{e}\left(p^{2}\right)^{\alpha-2}$ \\
\hline$f_{5}$ & & & $(\underline{2})-(\sqrt{ } t) t B_{f}^{*} B_{e} p^{2 \alpha-3}$ \\
\hline$f_{3}$ & (1) $t B_{y} * B_{y}\left(p^{2}\right)^{\alpha-1}$ & & $(\underline{3})-t B_{e}^{*} B_{e}\left(p^{2}\right)^{\alpha-2}$ \\
\hline$f_{1}$ & & (1) $-t B_{x} B_{x}^{*}\left(p^{2}\right)^{\alpha-1}$ & \\
\hline
\end{tabular}

If a residue can be obtained only by factorization, its phase is in many cases undetermined. We have not indicated such possible phase ambiguities in the Tables. $\mathrm{b}$ The residues of the leading $P=(-1)^{J}$ trajectory in (the $\pi \pi \rightarrow V V$ amplitudes) $\bar{f}_{11^{+-}}$and $\bar{f}_{11^{-}-}$are both $-(1 / t) B_{r}^{*} B_{r}\left(q^{2}\right)^{\alpha-1}$, with energy dependence
$\left(s / q^{2}\right)^{\alpha-1}$ and $\left(s / q^{2}\right)^{\alpha-2}$, respectively.

prevents analyticity from completely fixing the residue of the first daughter.

Notice that it frequently happens in these three reactions that conspiracy, analyticity, and factorization are not enough to completely determine the firstdaughter contribution in terms of the parent contribution. This seems to be due to the fact that the parent evades in so many of the reactions. In all cases, however, factorization of the daughter residues is consistent with the required analyticity properties of the amplitudes. This agrees with the notion that the contribution of a single Toller pole is composed of an infinite sum of factorizing Regge trajectories. ${ }^{41}$

We conclude that the analyticity and $O(4)$-symmetry approaches complement each other and agree when they overlap. For equal-mass scattering, when analyticity

${ }^{41}$ Note that for the examples we have chosen, all information about the second and lower daughters is obtained from singularity cancellation and factorization (as opposed to conspiracy). In $\mathrm{EE}$ reactions, no information may be obtained from analyticity arguments. Hence we always have the freedom to force factorization of these lower daughters. Combining this with the study in Tables II-VII (which demonstrates that the first daughters factorize), we conclude that factorization of all daughters is consistent with the analyticity constraints. tells us little, the $O(4)$ analysis gives much stronger results because of the $O(4)$ invariance of the scattering amplitude. The unequal-mass reactions with spin, which are difficult to treat from a group-theoretical viewpoint, are the ones which provide the most information when analyticity is involved. For Regge-pole models, in which one would like to invoke the additional dynamical postulate of factorization, the combination of this with analyticity considerations can then give information about equal-mass scattering and and also about cases in which the parent residue evades. Hence the joint requirements of analyticity and factorization enable one to specify many features of any strong-interaction theory.

Note added in proof. The use of factorization to determine the equal mass residues of daughter trajectories, as described above, has been worked out in detail for the spinless case by J. B. Bronzan and C. E. Jones [Phys. Rev. Letters 21, 564 (1968)]. Application of the analyticity (singularity-cancellation) approach to derive relations between slopes of parent and daughter trajectories, i.e., "mass formulas," has been investigated by several authors: J. Bronzan, C. E. Jones, and P. K. Kuo, Phys. Rev. (to be published); 
P. Di Vecchia and F. Drago, Frascati report, 1968 (unpublished); P. K. Kuo and J. F. Walker, Phys. Rev. (to be published).

Arguments favoring the $M=0$ pion type discussed in Sec. VI and Table VI have been given by R. F. Sawyer, Phys. Rev. Letters 21, 764 (1968).

\section{ACKNOWLEDGMENT}

The authors would like to acknowledge the participation of Professor Steven Frautschi during the early stages of this work.

\section{APPENDIX A : PROPERTIES OF $\boldsymbol{e}$ FUNCTIONS}

We use the functions defined in Ref. 18:

$$
\begin{aligned}
e_{\lambda \mu}{ }^{J}(z) & \equiv\left(\sqrt{2} \cos \frac{1}{2} \theta\right)^{-|\lambda+\mu|}\left(\sqrt{2} \sin \frac{1}{2} \theta\right)^{-|\lambda-\mu|} d_{\lambda \mu}{ }^{J}(\theta) \\
& =e_{\lambda \mu}{ }^{J+}+e_{\lambda \mu}{ }^{J-},
\end{aligned}
$$

where $e_{\lambda \mu}{ }^{J \pm}$ have opposite behavior under parity $(\theta \rightarrow \pi-\theta, z \rightarrow-z)$

with

$$
e_{\lambda \mu}^{J \pm}(-z)= \pm(-1)^{J-\Lambda} e_{\lambda \mu}{ }^{J \pm}(z)
$$

$$
\Lambda=\max (|\lambda|,|\mu|) \text {. }
$$

From $e_{\lambda-\mu}{ }^{J \pm}(z)= \pm(-1)^{\lambda+\Lambda} e_{\lambda \mu}{ }^{J \pm}(z)$, it follows that

$$
e_{\lambda 0}^{J-}=0 \text {. }
$$

The $e$ functions are related to Jacobi polynomials (up to a sign) as follows:

$$
\begin{array}{r}
e_{\lambda \mu}^{J \pm}=2^{-\Lambda-1}\left(\frac{(J+\Lambda) !(J-\Lambda) !}{(J+N) !(J-N) !}\right)^{1 / 2}\left[P_{J-\Lambda}^{(|\lambda-\mu|,|\lambda+\mu|)}\right. \\
\left. \pm(-1)^{\lambda+\Lambda} P_{J-\Lambda}^{(|\lambda+\mu|,|\lambda-\mu|)}\right],
\end{array}
$$

where $N=\min (|\lambda|,|\mu|)$ and the Jacobi polynomials have normalization

$$
P_{n}{ }^{(a, b)}(z=1)=\left(\begin{array}{c}
n+a \\
n
\end{array}\right) .
$$

In order to expand $e^{J \pm}$ in powers of $z$, we use

$$
\begin{aligned}
P_{J}(z) & =\frac{2^{J} \Gamma\left(J+\frac{1}{2}\right)}{\Gamma(J+1)}\left(z^{J}-\frac{J(J-1)}{2(2 J-1)} z^{J-2}+\frac{J(J-1)(J-2)(J-3)}{2 \times 4 \times(2 J-1)(2 J-3)} z^{J-4}+\cdots\right) \\
& =\frac{2^{J} \Gamma\left(J+\frac{1}{2}\right)}{\Gamma(J+1)} \sum_{k=0,2,4, \cdots}\left(\frac{(-1)^{k / 2} J(J-1) \cdots(J-k+1) z^{J-k}}{[2 \times 4 \times \cdots \times k][(2 J-1)(2 J-3) \cdots(2 J-k+1)]}\right)
\end{aligned}
$$

where we have neglected the other branch of the Legendre function for nonintegral $J$. (If we used the Mandelstam form of the Sommerfeld-Watson Reggeization, we would be using $E$, and not $e$, functions. See Appendix B of Ref. 18.) For $J$ nonintegral, the sum in Eq. (A5) does not terminate.

Using (A5), we can calculate the expansions needed in the processes $\pi \pi \rightarrow V V, \pi N \rightarrow V N$, and $N N \rightarrow N N$, where $\lambda, \mu=0,1$ :

$$
\begin{aligned}
& e_{00}{ }^{\alpha+}=e_{00}{ }^{\alpha}=P_{\alpha} \rightarrow \frac{2^{\alpha} \Gamma\left(\alpha+\frac{1}{2}\right)}{\Gamma(\alpha+1)} \\
& \times\left(z^{\alpha}-\frac{\alpha(\alpha-1)}{2(2 \alpha-1)} z^{\alpha-2}+\cdots\right), \\
& e_{00}{ }^{\alpha-}=0, \\
& e_{01}{ }^{\alpha+}=-e_{10}{ }^{\alpha+}=\frac{P_{\alpha^{\prime}}}{[\alpha(\alpha+1)]^{1 / 2}} \rightarrow \frac{2^{\alpha} \Gamma\left(\alpha+\frac{1}{2}\right)}{[\alpha(\alpha+1)]^{1 / 2} \Gamma(\alpha+1)} \\
& \cdot \times\left(\alpha z^{\alpha-1}-\frac{\alpha(\alpha-1)(\alpha-2)}{2(2 \alpha-1)} z^{\alpha-3}+\cdots\right), \\
& e_{01}{ }^{\alpha-}=0, \\
& e_{11}^{\alpha+}=\frac{P_{\alpha}^{\prime}+z P_{\alpha}^{\prime \prime}}{\alpha(\alpha+1)} \rightarrow \frac{2^{\alpha} \Gamma\left(\alpha+\frac{1}{2}\right)}{\alpha(\alpha+1) \Gamma(\alpha+1)} \\
& \times\left(\alpha^{2} z^{\alpha-1}-\frac{\alpha(\alpha-1)(\alpha-2)^{2}}{2(2 \alpha-1)} z^{\alpha-3}+\cdots\right),
\end{aligned}
$$

$$
\begin{aligned}
e_{11}^{\alpha-}=\frac{-P_{\alpha^{\prime \prime}}^{\prime \prime}}{\alpha(\alpha+1)} & \rightarrow \frac{2^{\alpha} \Gamma\left(\alpha+\frac{1}{2}\right)}{\alpha(\alpha+1) \Gamma(\alpha+1)}\left(\alpha(\alpha-1) z^{\alpha-2}\right. \\
& \left.-\frac{\alpha(\alpha-1)(\alpha-2)(\alpha-3)}{2(2 \alpha-1)} z^{\alpha-4}+\cdots\right) .
\end{aligned}
$$

\section{APPENDIX B : DAUGHTERS IN EU SCATTERING} $(\lambda=u=1)$

In this appendix we show for the EU case that daughterization (i.e., removal of undesired singularities by inclusion of single-parity daughter contributions) of the minor amplitude $\sum(2 J+1) T_{c d, a b}{ }^{J, P} e_{\lambda \mu}{ }^{J^{-}}$ensures daughterization of the major amplitude $\sum(2 J+1)$ $\times T_{c d, a b}{ }^{J, P} e_{\lambda \mu}{ }^{J^{+}}$(or vice versa). For this purpose, it is most convenient to absorb functions of $J$ into the partial-wave amplitudes and to reexpress (using Appendix A) the major and minor amplitudes, respectively, as

$$
\text { and } \begin{aligned}
& \sum T_{c a, a b}{ }^{J, P}\left[P_{J-\Lambda}{ }^{(\eta, \theta)}(z)+P_{J-\Lambda}^{(\theta, \eta)}(z)\right] \\
& \sum T_{c a, a b}{ }^{J, P}\left[P_{J-\Lambda}{ }^{(\eta, \theta)}(z)-P_{J-\Lambda}{ }^{(\theta, \eta)}(z)\right],
\end{aligned}
$$

where $\eta=|\lambda-\mu|, \quad \theta=|\lambda+\mu|, \lambda=a-b, \mu=c-d$, and $\Lambda=\max (|\lambda|,|\mu|)$. A pole in one integer spin state (at $J=\Lambda+n)$ would then produce a major amplitude of the form

$$
T_{c d, a b}{ }^{n^{\prime}}\left[P_{n}{ }^{(\eta, \theta)}(z)+P_{n}^{(\theta, \eta)}(z)\right]
$$


(for $n^{\prime}=n+\Lambda$ ). We will deal in this appendix only with integer $n$ and with the special values $\lambda=\mu=1 \quad \eta=0$, $\theta=2$ ), but the method can be extended to other cases. Expanding in powers of $z$ (since $z$ goes like $\sqrt{ } t)$, one has for integer $n$

$$
\begin{aligned}
& P_{n}^{(\eta, \theta)}(z)=\sum_{\nu=0}^{n} z^{\nu} g(\eta, \theta, \nu, n), \\
& P_{n}^{(\theta, \eta)}(z)=\sum_{\nu=0}^{n} z^{\nu} g(\theta, \eta, \nu, n),
\end{aligned}
$$

where the Jacobi polynomials have the symmetry

$$
P_{n}^{(\eta, \theta)}(-z)=(-1)^{n} P_{n}^{(\theta, \eta)}(z),
$$

which implies

$$
(-1)^{n-v} g(\eta, \theta, \nu, n)=g(\theta, \eta, \nu, n) .
$$

Thus we may represent the contribution of the leading pole and all of its even daughters to the major amplitude by

$$
\begin{aligned}
& \sum_{\sigma=0}^{[n]} T^{n^{\prime}-\sigma}\left[P_{n-\sigma}{ }^{(\eta, \theta)}(z)+P_{n-\sigma}{ }^{(\theta, \eta)}(z)\right] \\
& =\sum_{\sigma=0}^{[n]} T^{n^{\prime}-\sigma} \sum_{\nu=[0]}^{n-\sigma} 2 z^{\nu} g(\eta, \theta, \nu, n-\sigma)
\end{aligned}
$$

(we use $[n]$ to represent the largest even integer smaller than $n$, and [0] to represent the smallest integer allowed in the sum, given the restrictions on $\nu$ and $\sigma$ ), where $\sigma$ is restricted to be even (only even daughters are needed) and $n-\sigma-\nu$ is also even (other terms cancel in summing the two Jacobi polynomials). The contribution to the minor amplitude then becomes

$$
\begin{aligned}
\sum_{\sigma=0}^{[n]} T^{n^{\prime}-\sigma} & {\left[P_{n-\sigma}(\eta, \theta)(z)-P_{n-\sigma}{ }^{(\theta, \eta)}(z)\right] } \\
& =\sum_{\sigma=0}^{[n]} T^{n^{\prime}-\sigma} \sum_{\nu=[0]}^{n-\sigma} 2 z^{\nu-1} g(\eta, \theta, \nu-1, n-\sigma),
\end{aligned}
$$

where $\sigma$ and $n-\sigma-\nu$ are again even.

As pointed out in Sec. II, we need only consider the most singular terms in the daughter residue in this proof (the proof for less singular pieces then follows); it was also shown there that the most singular pieces of parent and daughter residues all have the same behavior near $t=0$, viz.,

$$
T^{n^{\prime}-\sigma} \rightarrow \gamma^{(\sigma)}(p q)^{n-\sigma} \sim \frac{\text { (const) }}{t^{\sigma / 2} t^{(n-\sigma) / 2}}=\frac{C_{n^{\prime}-\sigma}}{t^{n / 2}} .
$$

Because $z$ behaves like $\sqrt{ } t$ near $t=0$ for the EU case, the requirement that the minor amplitude have no unwanted singularities at $t=0$ may be restated as

$$
\sum_{\sigma=0}^{[n-\nu]} C_{n^{\prime}-\sigma g}(0,2, \nu-1, n-\sigma)=0
$$

for even $\sigma$ and all $\nu \leq n-2$, with $n-\sigma-\nu$ even. Likewise, the condition for daughterization of the major amplitude is

$$
\sum_{\sigma=0}^{[n-\nu]} C_{n^{\prime}-\sigma g} g(0,2, \nu, n-\sigma)=0
$$

with the same constraints on the indices. To be more exact, these conditions are those which pertain to unnatural-parity exchange in $\pi N \rightarrow V N$; the major and minor residues for natural-parity exchange may be obtained, respectively, by multiplying the major and minor residues obtained here by $\sqrt{ } t$.

With the replacement $l=n-\nu$ for $l$ even, we can rewrite (B5) in the form

$$
\sum_{\sigma=0}^{[l]} C_{n^{\prime}-\sigma} g(0,2, n-l-1, n-\sigma)=0,
$$

and (B6) in the form

$$
\sum_{\sigma=0}^{[l]} C_{n^{\prime}-\sigma} g(0,2, n-l, n-\sigma)=0 .
$$

From the functions in Appendix A, one can deduce that

$$
\frac{g(0,2, n-\sigma-l, n-\sigma)}{g(0,2, n-\sigma-l-1, n-\sigma)}=-\left(\frac{n-\sigma-l+1}{n-\sigma-l}\right)
$$

for all $l$ such that $n-\sigma-l>0$, i.e.,

$$
\frac{g(0,2, n-x, n-\sigma)}{g(0,2, n-x-1, n-\sigma)}=-\left(\frac{n-x+1}{n-x}\right) .
$$

Hence,

$$
\begin{aligned}
\sum_{\sigma=0}^{[l]} C_{n^{\prime}-\sigma g}(0,2, n-l-1, n-\sigma) \\
=-\left(\frac{n-l}{n-l+1}\right) \sum_{\sigma=0}^{[l]} C_{n^{\prime}-\sigma g}(0,2, n-l, n-\sigma)
\end{aligned}
$$

and the one set of conditions for regularization by daughters implies the other.

\section{APPENDIX C: DAUGHTERS IN UU SCATTERING $(\lambda=u=1)$}

Using the same notation as in Appendix B, we prove in this Appendix that for a UU process the regularization of $\tilde{f}_{10,10} \sim T_{10,10}{ }^{\alpha} P_{\alpha-1}{ }^{(0,2)}$ by including even and odd daughters,

$$
T_{10,10^{\alpha}} P_{\alpha-1}^{(0,2)}(z) \rightarrow \sum_{\sigma} T_{10,10^{\alpha-\sigma}}(t) P_{\alpha-\sigma-1}{ }^{(0,2)}(z)
$$


(so that the result behaves like a constant near $t=0$ ), ensures the regularization of $\bar{f}_{10,-10} \sim T_{10,10} \alpha P_{\alpha-1}(2,0)$ by

$$
\sum_{\sigma} T_{10,10^{\alpha-\sigma}(t) P_{\alpha-\sigma-1}(2,0)}(z)
$$

(i.e., this latter amplitude then behaves like $1 / t$ near $t=0)$. Again we give the proof for integer spin only.

As shown in Eq. (2.29), $z-1$ behaves like $t$ near $t=0$ for $\Delta_{a c} \Delta_{b d}>0$. It is therefore convenient to use, as a representation for the Jacobi polynomial, ${ }^{42}$

$$
P_{n}^{(\theta, \eta)}(z)=\sum_{\nu=0}^{n}\left(\begin{array}{c}
\theta+n \\
\theta+\nu
\end{array}\right)\left(\begin{array}{c}
n+\theta+\eta+\nu \\
n+\theta+\eta
\end{array}\right)\left(\frac{z-1}{2}\right)^{\nu} .
$$

Hence,

$$
P_{n}^{(\theta, \eta)}(z)=P_{n}^{(\eta, \theta)}(z)+f_{n}^{\eta, \theta}(z),
$$

where

$$
\begin{aligned}
f_{n}^{\eta, \theta}(z)=\sum_{j=0}^{\theta-\eta-1}\left(\begin{array}{c}
\theta-\eta \\
j
\end{array}\right) \sum_{\nu=0}^{n-1}\left(\begin{array}{c}
\eta+n \\
\theta+\nu-j
\end{array}\right) \\
\times\left(\begin{array}{c}
n+\eta+\theta+\nu \\
\nu
\end{array}\right)\left(\frac{z-1}{2}\right)^{\nu}
\end{aligned}
$$

(we assume $\theta>\eta$ for this formula).

Following the discussion in Sec. II, the daughter residues are chosen such that

$$
\sum_{\sigma=0}^{n} T_{10,10^{n^{\prime}-\sigma} P_{n-\sigma}(0,2)}
$$

is regular at $t=0$. It was shown there that the most singular pieces have the behavior $T^{n^{\prime}-\sigma} \sim\left(C_{n^{\prime}-\sigma} / t^{n}\right)$ where $C_{n^{\prime}-\sigma}$ is a constant. Then

$$
\sum_{\sigma=0}^{n} T_{10,10^{n^{\prime}}-\sigma} \sum_{\nu=0}^{n-\sigma}\left(\begin{array}{c}
n-\sigma \\
\nu
\end{array}\right)\left(\begin{array}{c}
n-\sigma+2+\nu \\
n-\sigma+2
\end{array}\right)\left(\frac{z-1}{2}\right)^{\nu}
$$

$\sim$ const $\quad(\mathrm{C} 4)$

implies

$$
\begin{array}{r}
\sum_{\nu=0}^{n}\left(\frac{z-1}{2}\right)^{\nu} \sum_{\sigma=0}^{n-\nu} T_{10,10^{n}-\sigma}\left(\begin{array}{c}
n-\sigma \\
\nu
\end{array}\right)\left(\begin{array}{c}
n-\sigma+2+\nu \\
\nu
\end{array}\right) \\
\sim \text { const, }
\end{array}
$$

i.e., for $\nu \neq n$,

$$
\sum_{\sigma=0}^{n-\nu} C_{n^{\prime}-\sigma}\left(\begin{array}{c}
n-\sigma \\
\nu
\end{array}\right)\left(\begin{array}{c}
n-\sigma+2+\nu \\
\nu
\end{array}\right)=0 .
$$

The behavior of the associated amplitude $\vec{f}_{10,-10}$,

$$
\begin{aligned}
\sum_{\sigma=0}^{n} T_{10,10^{n^{\prime}-\sigma}} P_{n-\sigma}(2,0)(z)=\sum_{\sigma=0}^{n} T_{10,10^{n^{\prime}-\sigma} P_{n-\sigma^{(0,2)}}(z)} & \\
& +\sum_{\sigma=0}^{n} T_{10,10^{n^{\prime}-\sigma}} f_{n-\sigma^{0}}(z),
\end{aligned}
$$

42 G. Szego, Orthogonal Polynomials (Edwards Brothers, Inc., Ann Arbor, Mich., 1948). depends on that of

$$
\begin{array}{r}
\sum_{\sigma=0}^{n} T_{10,10^{n^{\prime}-\sigma}} f_{n-\sigma^{0,2}}(z)=\sum_{\nu=0}^{n-1}\left(\frac{z-1}{2}\right)^{\nu} \sum_{\sigma=0}^{n-\nu-1} T_{10,10^{n^{\prime}-\sigma}} \\
\times\left(\begin{array}{c}
n-\sigma+2+\nu \\
\nu
\end{array}\right) \sum_{j=0}^{1}\left(\begin{array}{l}
2 \\
j
\end{array}\right)\left(\begin{array}{c}
n-\sigma \\
2+\nu-j
\end{array}\right) .
\end{array}
$$

Again, $t=0$ behavior is governed by the coefficients of $(z-1)^{\nu}$. For $\nu \neq n-1$, the coefficient

$$
\sum_{\sigma=0}^{n-\nu-1} T_{10,10^{n^{\prime}-\sigma}}\left(\begin{array}{c}
n-\sigma+2+\nu \\
\nu
\end{array}\right) \sum_{j=0}^{1}\left(\begin{array}{l}
2 \\
j
\end{array}\right)\left(\begin{array}{c}
n-\sigma \\
2+\nu-j
\end{array}\right)
$$

can be put into the form

$$
\begin{array}{r}
\sum_{\sigma=0}^{n-\nu^{\prime}} T_{10,10^{n^{\prime}-\sigma}} \frac{(n-\sigma) !}{(n-\sigma+2) !} \frac{\left(n-\sigma+\nu^{\prime}+2\right) !}{\left(n-\sigma-\nu^{\prime}\right) !\left(\nu^{\prime}-1\right) !\left(\nu^{\prime}+1\right) !} \\
\text { where } \quad \nu^{\prime}<n . \quad \text { (C7) }
\end{array}
$$

However, the conditions for regularization by daughters are [Eq. (C5)]

$$
\sum_{\sigma=0}^{n-\nu} C_{n^{\prime}-\sigma} \frac{(n-\sigma) !}{(n-\sigma+2) !} \frac{(n-\sigma+\nu+2) !}{(n-\sigma-\nu) ! \nu ! \nu !}=0 \quad \nu<n .
$$

Thus (C7) is also zero, and the only contribution to (C6) behaves like $(z-1)^{n-1} T^{n^{\prime}} \sim 1 / t$ near $t=0$. Thus we have shown

$$
\sum_{\sigma=0}^{n} T_{10,10^{n^{\prime}-\sigma}} f_{n-\sigma^{0,2}}(z) \sim 1 / t .
$$

\section{APPENDIX D : PERTURBATION-THEORY EXAMPLES}

During the course of this work, we have found the study of individual Feynman graphs to be extraordinarily helpful. ${ }^{43}$ Because each Feynman graph is Lorentzinvariant, it must have the proper singularity structure. Thus the singularity cancellation between parents and daughters may be made manifest simply by decomposing the calculated helicity amplitudes into a sum of the appropriate Jacobi polynomials. The daughters are particularly obvious in our examples because they are nonpole contributions to the graph.

Unfortunately, many graphs seem to contain contributions from more than one Toller pole at $t=0$, especially in nonleading powers of $s$. We do not wish to include in this paper a complete theory of Feynman graphs in terms of Toller poles ${ }^{44}$; therefore we have

${ }^{43}$ See also L. Durand, Phys. Rev. Letters 18, 58 (1967); R. Blankenbecler and R. Sugar, Phys. Rev. 168, 1597 (1968); R Blankenbecler, R. Sugar, and J. Sullivan, ibid. 172, 1451 (1968); R. L. Sugar and J. D. Sullivan, ibid. 166, 1515 (1968).

${ }^{44}$ This may be done using the technique of R. F. Sawyer, Phys. Rev. 167, 1372 (1968). 
chosen to present a series of graphs which demonstrate the particular singularity structures discussed in the text and to explain them in terms of the Toller poles they do contain. This exercise is doubly gratifying: It is amusing to see how the diagrams reproduce in a particular case for integer spin the results derived from more general considerations, but it is also handy when calculating the diagrams for other purposes to be able to check the results. Without further excuse, therefore, we present some examples.

First, consider the pattern of singularity cancellation in a UU process like $\pi \pi \rightarrow V V$. For the two possible types of single-parity exchange, we calculate spin-2 exchange (which is the lowest spin to show daughters in the helicity-flip-1 amplitude).

For $2^{+}$exchange in $\pi \pi \rightarrow V V$, one may take the amplitude

$$
\epsilon^{\mu \alpha \beta \gamma} \epsilon_{1}^{\beta} q_{1}{ }^{\gamma} k_{1}{ }^{\alpha}\left(k_{1}-q_{1}\right)^{\sigma} P_{\mu \sigma^{\nu}} \epsilon^{\nu \delta z \theta} \epsilon_{2}{ }^{\delta} q_{2}{ }^{z} k_{2}{ }^{\theta}\left(k_{2}-q_{2}\right)^{\tau}
$$

where $k_{i}$ are the momenta of the vector mesons involved, $\epsilon_{i}$ are their wave functions, and $q_{i}$ are the momenta of the pions. If one uses for $P_{\mu \sigma}{ }^{\nu \tau}$ the $2^{+}$propagator given by Durand, ${ }^{43}$ this results in

$$
\begin{aligned}
\left(t-m_{a}^{2}\right)\left(\bar{f}_{10,10}{ }^{t}-\bar{f}_{10,-10}{ }^{t}\right)=32 t q^{4} & \cos \theta_{t}-\left(4 q^{2} / m_{a}^{2}\right) \\
& \times\left(m_{V}{ }^{2}-m_{\pi}^{2}\right)^{2}\left(m_{a}^{2}-t\right),
\end{aligned}
$$$$
\left(t-m_{a}^{2}\right)\left(\bar{f}_{10,10}{ }^{t}+\bar{f}_{10,-10}\right)=-16 t q^{4}
$$

where $m_{a}$ is the mass of the exchanged particle and $q$ $=T_{V \pi} / 2 \sqrt{ } t$. Hence, the PCHA $\bar{f}_{10,10}{ }^{t}-\bar{f}_{10,-10}{ }^{t}$ behaves like $s^{1}=s^{\alpha-1}$ and all singularities at $t=0$ occur in lower powers of $s$. Notice that the "minor" amplitude contains a singularity like $1 / t$ which matches the one in the "major" amplitude, and that this one in the "major" amplitude arises from both the parent and the first daughter.

Similarly, a graph for $2^{-}$exchange is

This results in

$$
\epsilon_{1}{ }^{\mu} q_{1}{ }^{\sigma} P_{\mu \sigma}{ }^{\nu \tau} \epsilon_{2}{ }^{\nu} q_{2}{ }^{\tau} \text {. }
$$

$$
\begin{aligned}
& \left(t-m_{a}^{2}\right)\left(\bar{f}_{10,10^{t}}+\bar{f}_{10,-10^{t}}\right)=8 q^{2} \cos \theta_{t}-4 q_{0}^{2}\left(1-t / m_{a}^{2}\right), \\
& \left(t-m_{a}^{2}\right)\left(\bar{f}_{10,10}{ }^{t}-\bar{f}_{10,-10}\right)=-4 q^{2},
\end{aligned}
$$

which have properties similar to those noted above.

In contrast, one may consider a graph for paritydoubled exchange of the type given by Blankenbecler, Sugar, and Sillivan ${ }^{43}$ :

This gives

$$
\left[\epsilon^{1} \cdot k^{2} \epsilon^{2} \cdot k^{1}-\epsilon^{1} \cdot \epsilon^{2} k^{1} \cdot k^{2}\right] k^{1} \cdot k^{2} .
$$

$$
\begin{aligned}
\bar{f}_{10,10}+\bar{f}_{10,-10} & =k_{0}^{2}\left[-s+2 m_{V}^{2}\right], \\
\bar{f}_{10,10}-\bar{f}_{10,-10} & =-k^{2}\left[-s+2 m_{V^{2}}\right], \\
f_{00,00} & =\left(m_{V}{ }^{2} 2\right)\left[s / 2 k^{2}+1\right]\left[-s+2 m_{V}^{2}\right], \\
\bar{f}_{00,10} & =\left(k^{0} m_{V} / 2 \sqrt{2}\right)\left[-s+2 m_{V}^{2}\right],
\end{aligned}
$$

where $k=T_{V \pi} / 2 \sqrt{ } t$ and $k_{0}=\left(t+m_{V}^{2}-m_{\pi}^{2}\right) / 2 \sqrt{ } t$. This clearly demonstrates the equal and opposite singularities in $\bar{f}_{11}{ }^{+}$and $\bar{f}_{11}^{-}$multiplying $s^{\alpha-1}$. When the amplitudes are reexpressed in terms of Jacobi polynomials, it is found that the residues of the leading pole factorize at $t=0$ as shown in Table IV. As the nonleading terms arising from this graph contain $M=0$ Tollerpole pieces as well as the daughters of both poles, there is considerable complication in the lower powers of $s$ and we will not discuss them here.

If one looks instead at the EU reaction $\pi N \rightarrow V N$, one obtains from the graph $q^{\mu} \epsilon^{\sigma} P_{\mu \sigma}{ }^{\nu \tau} \bar{N} \gamma_{5} \gamma^{\nu} N P_{1}{ }^{\tau}$ for $2^{-}$ exchange ( $q^{\mu}$ is pion momentum; $P_{1}{ }^{\tau}$ is one of the nucleon momenta):

$\bar{f}_{10,1 / 2-1 / 2}{ }^{t}-\bar{f}_{10,-1 / 21 / 2} t=\frac{-2 p^{2} q \cos \theta_{t}}{\sqrt{2} M}$

$$
-\frac{P}{\sqrt{2} M}\left(t+m_{\pi}^{2}-m_{V}^{2}\right)\left(\frac{t}{m_{A}^{2}}-1\right),
$$

$f_{10,1 / 2-1 / 2}{ }^{t}+f_{10,-1 / 21 / 2} t=\frac{+p^{2} q}{\sqrt{2} M}$,

$\bar{f}_{10,1 / 21 / 2}-f_{10,-1 / 2-1 / 2}=\frac{p q_{0}}{\sqrt{2}}$

$$
\begin{array}{r}
\bar{f}_{00,1 / 21 / 2}=\frac{p \cos \theta_{t}}{4 m_{V}}\left[\frac{\left(m_{V}^{2}-m_{\pi}^{2}\right)^{2}}{t}-\left(m_{V}^{2}+m_{\pi}^{2}\right)\right] \\
-\frac{2 q E q^{0}}{3}\left(1-t / m_{A}^{2}\right)+\frac{E k^{0} q}{3 m_{V}}
\end{array}
$$

$$
\begin{aligned}
\bar{f}_{00,1 / 2-1 / 2}=-\frac{p^{2} q k_{0} \cos \theta_{t}}{M m_{V}} & +\frac{p E}{4 M m_{V}}\left(1-t / m_{A}^{2}\right) \\
\times & {\left[\frac{\left(m_{V}^{2}-m_{\pi}^{2}\right)^{2}}{t}-\left(m_{V}^{2}+m_{\pi}^{2}\right)\right] . }
\end{aligned}
$$

Here, $q$ and $k_{0}$ have the same definitions as above; $q_{0}=\sqrt{ } t-k_{0} ; E=\frac{1}{2} \sqrt{ } t ; P=\frac{1}{2}\left(t-4 M^{2}\right)^{1 / 2} ;$ and $M$ is the nucleon mass. Note that the "dominant" amplitude $\bar{f}_{11}-$ has no singularity at $t=0$, and behaves like $s^{1}=s^{\alpha-1}$. The "minor" amplitude $\bar{f}_{11}++$ does have a singularity at $t=0$, which conspires with $\bar{f}_{10}{ }^{-+}$. The conspiracy equation between $\bar{f}_{00}{ }^{-}$and $\bar{f}_{01}$ is also satisfied by conspiracy. For these, the subsidiary terms in $s$ conspire among themselves and again play a role different from that of the Toller pole discussed in the text, so we 
ignore them. The leading terms in these two satisfy conspiracy among themselves, paralleling the wellknown case of axial-vector exchange, which gives

$$
\begin{aligned}
f_{00,1 / 2-1 / 2} t & =\frac{\left(t+m_{V}^{2}-\mu^{2}\right)\left(t-4 M^{2}\right)^{1 / 2}}{4 \sqrt{ } t m_{V} M\left(t-m_{A}^{2}\right)}, \\
\tilde{f}_{00,1 / 21 / 2}{ }^{t} & =-\frac{\left\{\left[t-\left(m_{V}-\mu\right)^{2}\right]\left[t-\left(m_{V}+\mu\right)^{2}\right]\right\}^{1 / 2}}{(2 \sqrt{ } t) m_{V} m_{a}^{2}} .
\end{aligned}
$$

For the $M=1$ case, we need only cite the behavior of the nucleon Born-term graph in pion photoproduction
$\bar{N} \gamma_{5}(\gamma \cdot k)(\gamma \cdot \epsilon) N$. This gives (for a photon of mass $m_{V}$ )

$$
\begin{aligned}
\bar{f}_{00,++} & =-p m_{V}\left(\cos \theta_{t}\right) / M, \\
\bar{f}_{10,++}-\bar{f}_{-10,++} & =\sqrt{2} p k_{0} / M, \\
\bar{f}_{10,++}+\bar{f}_{-10,++} & =-\sqrt{2} E q / M, \\
\bar{f}_{00,+-} & =0, \\
\bar{f}_{10,+-}-\bar{f}_{-10,+-} & =\sqrt{2} q, \\
\bar{f}_{10,+-}+\bar{f}_{-10,+-} & =0,
\end{aligned}
$$

which show in a very simple way the singularities of the $M=1$ conspiracy discussed in Sec. V, even though the graph does not represent a $t$-channel exchange.

\title{
Currents as Coordinates in Nonrelativistic Quantum Mechanics*
}

\author{
W. J. Pardee, L. Schlessinger, and Jon Wright \\ Department of Physics, University of Illinois, Urbana, Illinois
}

(Received 28 June 1968)

\begin{abstract}
The formulation of nonrelativistic quantum mechanics using currents and densities as coordinates is investigated. A general solution for a single-particle theory is presented, and several many-body problems are discussed.
\end{abstract}

\section{INTRODUCTION}

$\mathbf{R}^{\mathrm{E}}$ ECENTLY, there has been considerable interest in the description of strong interactions in terms of currents. Dashen and Sharp ${ }^{1}$ showed that nonrelativistic quantum mechanics could be described by using currents and densities as coordinates rather than the more familiar canonical coordinates, but they left unanswered the question of solvability of such a theory. In this paper we treat systems of $Q$ identical bosons in their formalism, and show how to obtain all of the information that the usual formulation gives. For single particles interacting with a fixed potential or potential scattering of two particles, the new formalism turns out to require the solution of the Schrödinger equation. For more particles it is not clear what the form of the solution is except in some simple solvable examples.

It seems natural to formulate many-body problems in terms of currents and densities, and perhaps this approach might lead to better or different approximation schemes. Although our original motivation for solving problems this way was to learn how to work with descriptions of systems in terms of currents and densities, we also have shown that the formulation is a feasible approach to nonrelativistic problems. There is

* Work supported by the National Science Foundation under Grant No. NSF GP 6198 and the Office of Naval Research under Contract No. N00014-67-A-0305-0005.

${ }_{1}$ R. F. Dashen and D. H. Sharp, Phys. Rev. 165, 1857 (1968). no pretense made of mathematical rigor, and since we are in fact working with functional integrals-a relatively unexplored area of mathematics - we may occasionally adopt questionable mathematical procedures.

We start with a review of the work of Dashen and Sharp ${ }^{1,2}$ and refer the reader to their paper for further details. Section III is devoted to a discussion of a single particle interacting with a fixed potential, and there it is shown how to find solutions to the usual problems. In Sec. IV we treat noninteracting systems and show how to find the many-boson correlation functions. We also discuss the differences between wave functionals that give identical results for a single-particle theory but different results for a many-particle theory. The last section discusses two problems of interacting bosons-coupled harmonic oscillators and a one-dimensional system of particles interacting through $\delta$-function potentials. The form of the exact solutions to these problems suggests approximation schemes for other kinds of interactions, but it sheds no real light on how to obtain solutions to more complicated systems.

\section{REVIEW}

In this section we review for completeness the formulation of nonrelativistic quantum mechanics in terms of currents and charge densities as given by Dashen and

2 D. H. Sharp, Phys. Rev. 165, 1867 (1968).

${ }^{3} \mathrm{We}$ always assume that integration by parts is permissible and that the boundary terms vanish. 